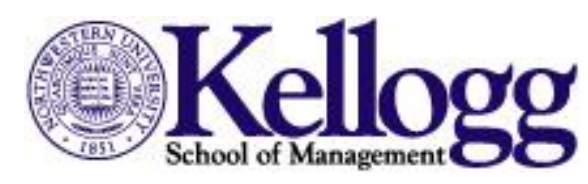

CMSEMS

Center for Mathematical Studies in Economics And Management Science Disc ussion Paper \#1553

\title{
AUCTIONS, ACTIONS, AND THE FAILURE OF INFORMATION AGGREGATION
}

\author{
Alp E. Atakan \\ Mehmet Ekmekci \\ Koc University \\ Northwestem University
}

October 10, 2012

J EL Classific a tion: C73, D44, D82, D83.

Keywords: Auctions, Large markets, Information Aggregation
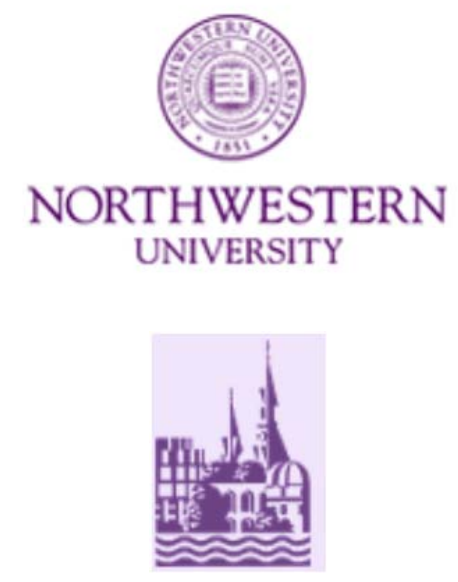


\title{
AUCTIONS, ACTIONS, AND THE FAILURE OF INFORMATION AGGREGATION
}

\author{
Alp E. Atakan And Mehmet EkmekCi
}

Date: October 10, 2012.

We study a market in which $k$ identical and indivisible objects are allocated using a uniform-price auction where $n>k$ bidders each demand one object. Before the auction, each bidder receives an informative but imperfect signal about the state of the world. The good that is auctioned is a common-value object for the bidders, and a bidder's valuation for the object is determined jointly by the state of the world and an action that he chooses after winning the object but before he observes the state. We show that there are equilibria in which the auction price is completely uninformative about the state of the world and aggregates no information even in an arbitrarily large auction. In the equilibrium that we construct, because prices do not aggregate information, agents have strict incentives to acquire costly information before they participate in the market. Also, market statistics other than price, such as the amount of rationing and bid distributions contain extra information about the state. Our findings sharply contrast with past work which shows that in large auctions where there is no ex-post action, the auction price aggregates information.

KEYWORDs: Auctions, Large markets, Information Aggregation

C73, D44, D82, D83.

Koç University, Department of Economics, Rumeli Feneri Yolu, Sarıyer 34450, Istanbul, Turkey. Email: aatakan@ku.edu.tr.

MEDS, Kellogg School of Management, Northwestern University, 2001 Sheridan Road, Evanston, IL 60208. Email: m-ekmekci@kellogg.northwestern. edu.

${ }^{1}$ We would like to thank Sneehal Banarjee, Wioletta Dziuda, Mike Fishman, Johannes Horner, Tai-Wei Hu, Nenad Kos, Qingmin Liu, Paul Milgrom, Nicola Persico, Ben Polak, Larry Samuelson, Andy Skryzpacz, Jeroen Swinkels, Robert Wilson, Charles Zheng, and seminar participants at Yale University, Koç University, Econometric Society 2012 Summer Meetings, Games World Congress 2012, Bielefelt University, and Kadir Has University for their useful comments. 
1 Introduction $\quad 1$

2 Model $\quad 6$

2.1 Signals . . . . . . . . . . . . . . . . . . . . . 8

2.2 The value function. . . . . . . . . . . . . . . . . . . . . . . . . 9

2.3 Strategies and equilibrium . . . . . . . . . . . . . . . . . 12

3 Large Markets and the Failure of Information Aggregation 13

3.1 Information aggregation. . . . . . . . . . . . . . . 13

3.2 Failure of information aggregation. . . . . . . . . . . . . . . 14

3.3 Sketch of the construction. . . . . . . . . . . . . . . . . . . 15

3.4 Properties of equilibrium. . . . . . . . . . . . . . . . . . . . . . . . . . . 19

3.5 Loser's curse. . . . . . . . . . . . . . . . . . . . . . . . . . . . . 21

4 Information Aggregation Failures in Monotone Equilibria 22

4.1 Indeterminate and suppressed prices. . . . . . . . . . . . . . . 27

5 Discussion $\quad 28$

5.1 Auctions with no Ex-Post Actions, Auctions with Actions, and Assumption 1. 28

5.2 Sketch of the Construction with Finite Markets. . . . . . . . . . . . . . . . 30

6 Conclusion $\quad 32$

$\begin{array}{ll}\text { A Organization of the Appendix } & 33\end{array}$

B Proof of Theorem 2 33

B.1 Method used for the construction . . . . . . . . . . . . . . . . . . 33

B.2 Step 1: Cutoff type . . . . . . . . . . . . . . . . . . . . . . 33

B.3 Setting the pooling bid and its properties . . . . . . . . . . . . . 35

B.4 Step 2: Checking deviations . . . . . . . . . . . . . . 36

B.4.1 Bidders with signals above $s_{z}^{p} \ldots \ldots$. . . . . . . . . . 36

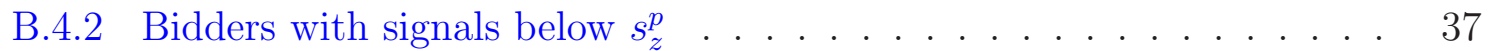

C Proof of Theorem $1 \quad 40$

D Proof of Lemma $1 \quad 41$

E Miscallenous Results 43 
"We must look at the price system as a mechanism for communicating information if we want to understand its real function.... The most significant fact about this system is the economy of knowledge with which it operates, or how little the individual participants need to know in order to be able to take the right action....by a kind of symbol, only the most essential information is passed on..." ?.

\section{INTRODUCTION}

One important reason to trust markets arises from the belief that market prices accurately summarize the vast array of information held by market participants. Whether this belief is justified, that is, whether prices efficiently aggregate information dispersed among agents that are active in an economy is a central economic question addressed by past research. In certain auction markets, prices do in fact effectively aggregate dispersed information. Specifically, consider a market in which a large number of identical common-value objects are sold through a uniform-price auction. In such an auction, if the bidders each have an independent signal about an unknown state of the world and if this unknown state determines the value of the object, then the equilibrium price converges to the true value of the object as the number of objects and the number of bidders grow arbitrarily large. Therefore, the auction price reveals information about the unknown state of the world. ?, ?, and ? have shown that this remarkable result holds under quite general assumptions.

In many situations, however, the common value of an object is not determined solely by the unknown parameters of the environment, i.e., the unknown state of the world. Rather, the object's value is also a function of how the object is utilized; in turn, the optimal way to utilize the object can depend on the unknown state of the world. For example, suppose that a large tract of land is to be divided and sold to farmers in smaller parcels through a uniform-price auction. Each farmer who successfully acquires a parcel of land in the auction needs to decide which crop to grow (e.g., wheat or rice). However, there is uncertainty about future crop prices as well as which crop grows best on that land. Alternatively, consider a uniform-price auction in which bandwidth is sold to telecommunication companies. Each winner must decide whether to use conventional technology or adopt an unconventional new one. However, there is uncertainty about future demand drivers (such as customer tastes) which will determine which technology is more profitable. In both of these examples, the winner of an object in the auction (a piece of land in the first and bandwidth in the second) must choose an action which will itself affect the value that the winner derives from the object. Moreover, this action must be taken after the auction is finalized but before some payoff-relevant uncertainty is resolved. ${ }^{1}$

\footnotetext{
${ }^{1}$ Numerous other auctions share the characteristics of the two that we highlight here. Examples include an auction for off-shore oil leases where the winner needs to undertake costly sunk investments in order to transform the oil reserves into productive use. However, exactly which sort of investment decision is wisest
} 
In the examples discussed above, if the auction price provides additional information that reduces uncertainty, i.e., if the auction price aggregates information, then the winners would make better decisions when choosing their action (which crop to grow or which technology to adopt). However, none of the past work on information aggregation in auctions explores how the information revealed by the auction price is used after the auction is completed. In contrast; in this paper we explicitly model how the information about the state of the world is used after a common-value auction is completed; in our model, the auction's winners must decide on an action in order to put the objects acquired into productive use and the optimal choice of action depends on the true state of the world. We show that such large common-value auctions have equilibria in which the equilibrium price reveals no information about the state of the world. Our result suggests that if information is useful for efficient decision making, then the equilibrium price may not aggregate all the information relevant for the decision. This finding stands in stark contrast to earlier studies which show that prices aggregate information if there is no immediate use for this information. ${ }^{2}$

More specifically, we study a model in which $k$ identical and indivisible objects are allocated using a uniform-price auction in which $n>k$ bidders each demand one unit of the good. Before the auction, each bidder receives an informative but imperfect signal about the state of the world. In the auction, bidders choose their bids as a function of their signal, the $k$ highest bidders are allocated one unit of the object, and all bidders who win an object pay a uniform price equal to the $k+1$ st highest bid. The good that is auctioned is a common-value object for the bidders and a bidder's valuation for the object is determined jointly by the state of the world and an action that he chooses after winning the object but before he observes the state. In a large market, if the market clearing price were to aggregate all information, then actions would be chosen efficiently and competition would necessarily drive the price of the object to its efficient-use value.

We explore a number of properties of markets as the numbers of bidders and the objects grow proportionately; however, our primary focus is on the informativeness of prices. An outsider who could observe the signals of an arbitrarily large number of bidders would learn the state of the world perfectly. Motivated by such an outsider's perspective, we say that prices fully aggregate information if an outsider can figure out the state of the world almost perfectly just by observing the equilibrium price of a large market.

may depend on parameters unknown to the market participants. Another example is an auction for iron ore where a winner must decide on which end product he will manufacture from the ore (e.g., flat steel versus steel rods). However, the ore's value to the winner will depend crucially on future end product prices and, therefore, the product mix that he chooses to manufacture.

${ }^{2}$ Although there is no allocative inefficiency in pure common-value environments, ? extend their first model to account for private values in addition to a common-value component, and show that uniform-price auctions achieve both allocative efficiency and informational efficiency. 
We present two main results. In our first main result, we construct a particular sequence of symmetric equilibria in which, as the market grows arbitrarily large, the limit price conveys no information about the true state of the world and remains strictly below the efficient usevalue of the object. Moreover, we show that such a sequence of equilibria can be constructed for a generic set of parameter values. In the equilibria we construct, a strictly positive fraction of agents chooses the wrong action because prices convey no new information. Therefore, inefficiency persists even in a large market whose outcome would have been efficient if one could observe all of the bidders' signals. Also, because the equilibrium price does not convey new information, agents have strict incentives to acquire costly information both before they participate in the auction and after the objects have been allocated.

A prominent property of the equilibrium which we construct is that equilibrium bids are nondecreasing in the signal that an agent receives, i.e., the bidding function is nondecreasing. In order to explore the robustness of our first result, we also study arbitrary symmetric equilibria in which the bidding function is monotonic. In our second result, we characterize equilibrium behavior in any symmetric equilibrium in which the bidding function is monotonic and we use this characterization to show that no sequence of such equilibria can fully aggregate information. In any symmetric equilibria where the bidding function is monotonic, the price fails to aggregate information and remains below the efficient use-value of the object.

To understand the logic of our findings, it is useful to further discuss the particular monotone equilibrium that we construct. In this equilibrium, bidders face two countervailing incentives that jointly determine equilibrium bids and thus the equilibrium price. The first is an incentive to compete: if a bidder makes strictly positive profits when he wins an object at a price equal to his bid, then he prefers to increase his bid. The second is an incentive to learn: a bidder would ideally like to know the signals of all the other bidders in the event that he wins an object and therefore has an incentive to bid strategically in order to acquire information.

In the particular monotone equilibrium that we construct, information is not aggregated by the price because of the existence of pooling. Pooling by bidders with a range of different signals at a certain pooling bid makes the equilibrium price less sensitive to the information of the bidders and thus leads to limited learning. In our construction, the pooling bid is sustained because of the incentive to learn and in spite of the incentive to compete. Specifically, when the price is equal to the pooling bid, objects are allocated using rationing among the bidders who choose the pooling bid. A bidder who chooses the pooling bid and wins an object through rationing at a price equal to the pooling bid obtains more information about the state of the world, compared to the case in which he instead chooses a higher bid, avoids rationing, and 
wins an object. This is because winning an object when rationing is applied is more likely in one state than the other. In other words, rationing is a lottery whose odds depend on the state of the world. If a bidder who chooses the pooling bid increases his bid, then he acquires an object more frequently because he avoids rationing when the price is equal to the pooling bid. However, in this case he forgoes the extra piece of information that comes from winning under rationing. Because this extra piece of information is sufficiently valuable for bidders who choose the pooling bid, these bidders refrain from increasing their bid even though they make strictly positive profits at the pooling bid.

In the paper, we also characterize all symmetric equilibria that are monotonic and we argue that these equilibria are qualitatively similar to the particular equilibrium discussed above. More specifically, we show that in all weakly increasing equilibria, players who receive low signals choose a pooling bid, players who receive high signals bid above the pooling bid, and the bidding function is strictly increasing above the pooling bid. Moreover, we show that prices can never fully aggregate information in monotone equilibria. That is, prices either do not reveal any information about the state of the world, or there is price indeterminacy, i.e., prices are not completely determined by the state of the world.

In a nutshell, our results suggest that the auction price may not be a very good aggregator of information if the information content of this price is needed to make decisions that affect the value of the objects. In our model, market statistics other than price, such as the amount of rationing and bid distributions, are informative. Therefore, whether these statistics are observed after an auction is finalized can affect how much information is aggregated by prices. ${ }^{3}$ Moreover, dynamic models in which traders engage in multiple rounds of activities may augment the accumulation of useful information.

Relation to the literature. This paper is related to earlier work which studies information aggregation in auction markets with indivisible goods, in large double auctions, in markets for divisible goods, in search markets, and in rational-expectation equilibria.

? studied second-price auctions with common value for one object for sale, and ? extended the analysis to any arbitrary number of objects. Both of these papers show that as the number of bidders gets arbitrarily large, price converges to the true value of the object, but only provided that there are bidders with arbitrarily precise signals about the state of the world. ? further generalize the previous analysis to the case where there are no arbitrarily precise signals. They show that prices converge to the true value of a common-value object in all symmetric equilibria if and only if both the number of identical objects and the number of bidders who are not allocated an object grow without bound. ? generalize the analysis

\footnotetext{
${ }^{3}$ This is still an open and interesting question. The transparency of the market changes the incentives of the bidders at the first place, it may cause them to conceal their own information.
} 
in ? to a mixed private, common-value environment. Finally, ? shows that the information aggregation properties of auctions are more general than the particular mechanisms studied before; he does this by providing a unified approach that uses the statistical properties of certain order statistics. ${ }^{4,5}$ The model that we present in this paper is closest to ?. The main difference from theirs is that in our model the object's value is jointly determined by the unknown state of the world and the action that the owner of the object later takes.

The information aggregation properties of prices in large double auctions is addressed in work by ? and ?. These papers show that prices in double auctions aggregate information and therefore allocate objects efficiently. ? allow for interdependent values whereas ? study private-value environments. The approach in all of these papers differs from ours because in these papers, the state of the world determines the common-value component of the objects to the bidders and there is no ex-post action choice by the winners.

Information aggregation properties of markets in which the objects are divisible have also been studied. Most prominently, ? studies competitive supply-schedule games, and shows that as the number of firms gets large, equilibrium behavior converges to price-taking behavior. ? focus on a similar model that allows for heterogeneity in the traders' private information; they show that interdependent values may cause non-monotonicity in the price informativeness with respect to market size. In our environment, objects are not divisible and each bidder has a unit demand.

Studies have also been carried out on whether prices in economies in which agents need to match in order to engage in a trade converge to Walrasian prices as the search frictions disappear. These papers study a dynamic environment in which trade and learning occur over time. The classical papers in this literature include ?? while ? provides an extensive survey. The paper closest to ours is ?. In this paper, the authors also establish that there is an atom in the bid distribution of a common-value auction and, as in our model, it is the incentive to learn that sustains pooling at the atom. ${ }^{6}$

Our work also relates to the literature on costly information acquisition in rationalexpectations models, such as ?? and ?. These papers explain the conceptual difficulties

\footnotetext{
${ }^{4}$ See also ? for a calculation of the convergence rate of prices to the true value, and ?, which shows that the results of ? do not generalize once there are individualized prices, i.e., when one considers discriminatory price auctions.

${ }^{5}$ Also, see ? for related results on the properties of order statistics.

${ }^{6}$ Also see ? for a unified approach to when search markets with disappearing frictions exhibit Walrasian outcomes. More recent work by ? presents a search model in which a seller searches for a buyer who observes a private signal about the value of an object. Their paper argues that equilibrium prices depend critically on the tail properties of the signal distributions. In the context of financial markets, ? show that experimenting with small offers for divisible goods achieves information aggregation and efficient allocations. Also, ? shows that trading "separable" securities in a dynamic environment allows information that is dispersed among the traders to get aggregated.
} 
in interpreting prices as both allocation devices and information aggregators. Specifically, they argue that if consumers and producers need to undertake a costly activity in order to acquire information, then equilibrium prices cannot reveal the state of the world perfectly. Their reasoning is as follows: if prices were to reveal the state perfectly, then no agent would have an incentive to pay for information in the first place; but if no agent acquires information, then the prices cannot reveal the state as there is no information to aggregate. However, as was the case for auction markets, these papers do not explicitly consider how the information revealed by the market price could be used by the market participants once they have completed their trade in the market. In our model, since prices do not aggregate information, agents have a strict incentive to acquire information. This finding contrasts with the findings of ?, who argue that agents have no incentive to acquire information precisely because prices are so efficient in aggregating information.

Rationing in equilibrium is a distinct feature of our model. Equilibrium rationing also occurs in certain credit market models. For example, ? show that rationing is an equilibrium phenomenon in credit markets if there is either adverse selection or moral hazard. In this paper, equilibrium interest rates continue to remain depressed even though they do not clear the credit market. Rates remain lower than what is needed to clear the market either because higher rates lead safer types to drop out from the demand pool or, alternatively, because they result in more aggressive risk taking by the debtors. ${ }^{7}$ In our model, there is also asymmetric information among the bidders; however, the seller has no preferences over who gets the objects. Thus, in our setting rationing plays a different role: some buyers choose to be rationed in order to receive extra information about the state of the world.

Finally, our model is related to the game analyzed in ?. ? show that trade is possible between two agents with the same preferences if the value of the object traded is jointly determined by an unknown state of the world and an ex-post action that the eventual owner of the object will undertake. In their model, trade is precluded by a no-trade theorem without any ex-post action. It is the ex-post action and the consequent value of information that lead to the possibility of trade. Our model shares the feature that the eventual owner of an object undertakes an action once trading is complete. However, whereas they consider a bilateral bargaining framework and focus on the possibility of trade, we analyze an auction framework with a large number of strategic bidders and focus on information aggregation.

\section{MODEL}

We consider a sealed-bid, uniform-price auction. In this auction, there are $n$ bidders with unit demand and $k$ identical objects. We denote the ratio of objects to bidders (i.e., market

\footnotetext{
${ }^{7}$ See also the papers by ?? for why rationing disappears if the lenders can screen the borrowers with an additional instrument other than the interest rate.
} 
tightness) in this auction by $\kappa:=\frac{k}{n}<1$. The set of states of the world is $\Omega:=\{L, R\}$ and we denote a generic element of this set by $\omega$. The state of the world is drawn according to a common prior $\pi \in[0,1]$, where $\pi$ denotes the prior probability that the state is $R$, and $1-\pi$ denotes the prior probability that the state is $L$. Each bidder $i$ observes a private signal $s_{i}$ that belongs to the set of signals $S=[0,1]$, and submits a bid $b_{i} \in[0, \infty)$. Each of the $k$ highest bidders receives an object and is called a winner; all other bidders are called losers. ${ }^{8}$ Each winner pays a price $p$ which is equal to the $(k+1)^{s t}$ highest bid.

The payoff of a bidder who does not win an object is equal to zero. We assume that a bidder who wins an object must choose an action from a finite set of actions denoted by $A$. This action, together with the state of the world, determines the winner's valuation for the good. Although all our of arguments go through with an arbitrary, finite number of actions, to keep exposition simple, we assume that $A=\{l, r\}$. A winning bidder's payoff is jointly determined by the auction price $p$, the action that he chooses $a \in A$, and the state of the world $\omega \in \Omega$. In particular, we assume that a winning bidder's payoff is equal to $v(a, \omega)-p$, where the function $v(a, \omega)$ gives the winner's valuation for the object. In what follows, we assume, without loss of generality, that $v(r, R) \geq v(l, L)$ and we make the following main assumption:

Assumption 1 The valuation function satisfies the following two inequalities:

$$
\begin{aligned}
& v(l, L)>v(r, L), \\
& v(l, L)>v(l, R) .
\end{aligned}
$$

Note that if the valuation function does not satisfy inequality (1), then $r$ is a weakly dominant action. Also, if the valuation function does not satisfy inequality (2), then for any given action a bidder's valuation for the good is higher in state $R$ than in state $L$. In what follows, we normalize the valuation function such that

$$
v(l, R)=v(r, L)=0
$$

This normalization is without loss of generality if a valuation function satisfies Assumption 1. Under this normalization, Assumption 1 requires that $v(l, L)>0$, or in words, that the bidder's valuation for the good be positive if his action matches the state of the world, and his valuation for the good be zero if his action does not match the state of the world.

\section{REMARK 1 Assumption 1 is the substantive assumption which allows us to argue that}

\footnotetext{
${ }^{8}$ To rank bids that are tied, nature picks a ranking of bidders at random with each ranking equally likely.
} 
information is not aggregated in our model. The main implication of Assumption 1 which we use in many of our arguments is the fact that a bidder's expected valuation for the good is a nonmonotonic function of the probability that he assigns to state $R$. See section 2.2 and figure 1 for a more detailed discussion of this nonmonotonicity. In contrast, if either inequality (1) or (2) is not satisfied, then a bidder's expected valuation for the good is a monotonic function of the probability that he assigns to state $R$. In either of these two cases, ?'s findings apply and therefore information is aggregated in every symmetric equilibrium of a large market. We discuss this issue in more detail in section 5.1.

2.1. Signals The set of signals is $S:=[0,1]$, and the bidders' signals are independently and identically distributed conditional on the state of the world. Each bidder's signal distribution has a cumulative distribution function $F(. \mid w)$ with a density function $f(. \mid w)$ for each $w \in \Omega$. Assumption 2 (Weak MLRP) $\frac{f(s \mid R)}{f(s \mid L)} \geq \frac{f\left(s^{\prime} \mid R\right)}{f\left(s^{\prime} \mid L\right)}$ for $s>s^{\prime}$.

Since we make the MLRP assumption only in its weak form, our model is able to accommodate discrete signals. Moreover, since there are only two states of the world, this assumption is without loss of generality because we can ensure that it is satisfied by reordering the signals.

Assumption 3 (Limited individual information) There exists a number $\eta>0$ such that $\eta<f(s \mid w)<\frac{1}{\eta}$ for every $s \in S, w \in \Omega$.

This assumption requires that signals convey only a bounded amount of information. Hence, there is no bidder who possesses arbitrarily precise information based solely on the bidder's signal.

Assumption 4 (Informative signals exist) $\frac{f(0 \mid R)}{f(0 \mid L)}<1$ and the likelihood function $\frac{f(s \mid R)}{f(s \mid L)}$ is continuous at zero.

When taken together with the weak MLRP assumption, this assumption implies that the signal distributions are not identical across the two states of the world.

In what follows, we refer to the $m^{\text {th }}$ highest value among $n$ signals by $Y_{n}^{m}$. We define the unique signals $s_{R}^{\kappa} \in S$ and $s_{L}^{\kappa} \in S$ such that $F\left(s_{R}^{\kappa} \mid R\right)=1-\kappa$ and $F\left(s_{L}^{\kappa} \mid L\right)=1-\kappa$. Recall that $\kappa<1$ is the market tightness, i.e., the ratio of objects to bidders. Intuitively, in a large market there are as many bidders with signals above $s_{R}^{\kappa}$ as there are objects in state $R$. Therefore, if we were to allocate the objects to the bidders with higher signals first, then, in state $R$, the bidders who receive an object would be exactly those bidders whose signals exceed $s_{R}^{\kappa}$. 
2.2. The value function. In this section, we define a bidder's value as a function of his beliefs. If a bidder believes that the probability of state $R$ is $p$, then we say that the bidder's likelihood ratio is $\rho:=p /(1-p)$. In what follows, we will work with the likelihood ratio instead of working directly with beliefs for analytic convenience. ${ }^{9}$ Since the bidders have a common prior on the state of the world, and this prior assigns probability $\pi$ to state $R$, their prior likelihood ratio is $\rho_{0}:=\pi /(1-\pi)$. Below we introduce the value function $u$ which gives a bidder's expected valuation for an object as a function of the likelihood ratio $\rho$. In particular, let $u:[0, \infty] \rightarrow \mathbb{R}$ be the function defined by

$$
u(\rho)=\max _{a \in\{l, r\}}\left\{\frac{1}{\rho+1} v(a, L), \frac{\rho}{\rho+1} v(a, R)\right\} .
$$

This function gives the bidder's expected value for the object as a function of his beliefs about the state of the world, expressed as the relative likelihood ratio about the state of the world. Note that $u(0)=v(l, L)$ and $\lim _{\rho \rightarrow \infty} u(\rho)=v(r, R)$. Let $\rho^{*} \in(0, \infty)$ be the unique solution to the following equation:

$$
\frac{1}{\rho+1} v(l, L)=\frac{\rho}{\rho+1} v(r, R) .
$$

This cutoff is the likelihood ratio that makes a bidder indifferent between action $l$ and $r$. Such a cutoff always exists because of Assumption 1. In what follows, we extensively use the fact that the value function $u(\cdot)$ is strictly decreasing in the interval $\left[0, \rho^{*}\right]$ and strictly increasing in the interval $\left[\rho^{*}, \infty\right)$. See figure 1 for a depiction of the value function.

For a bidder who receives signal $s \in[0,1]$, we denote his likelihood ratio, slightly abusing notation, to be $\rho(s)$, defined as follows:

$$
\rho(s)=\frac{\pi}{1-\pi} \frac{f(s \mid R)}{f(s \mid L)}=\rho_{0} \frac{f(s \mid R)}{f(s \mid L)} .
$$

More generally, if the bidders behave according to some bidding strategy profile $b$ and a bidder arrives at an information set $\mathbf{I}$, then the likelihood ratio, conditional on $\mathbf{I}$, is denoted as $\rho_{b}(\mathbf{I})=\frac{\operatorname{Pr}_{b}(\mathbf{I} \mid R)}{\operatorname{Pr}_{b}(\mathbf{I} \mid L)}$, where $\operatorname{Pr}_{b}(\mathbf{I} \mid R)$ and $\operatorname{Pr}_{b}(\mathbf{I} \mid L)$ denote the probability of reaching information set $\mathbf{I}$ conditional on states $R$ and $L$, respectively.

REMARK 2 In the current framework with $A=\{l, r\}$, the ordering of the bidders according to their expected valuation can change with the arrival of new information. In order to see this, consider a situation where $\rho(0)>\rho^{*}$, as in figure 2. Moreover, for the sake of exposition,

\footnotetext{
${ }^{9}$ The whole analysis could be redone working directly with beliefs as there is a one-to-one mapping between likelihood ratios and beliefs.
} 


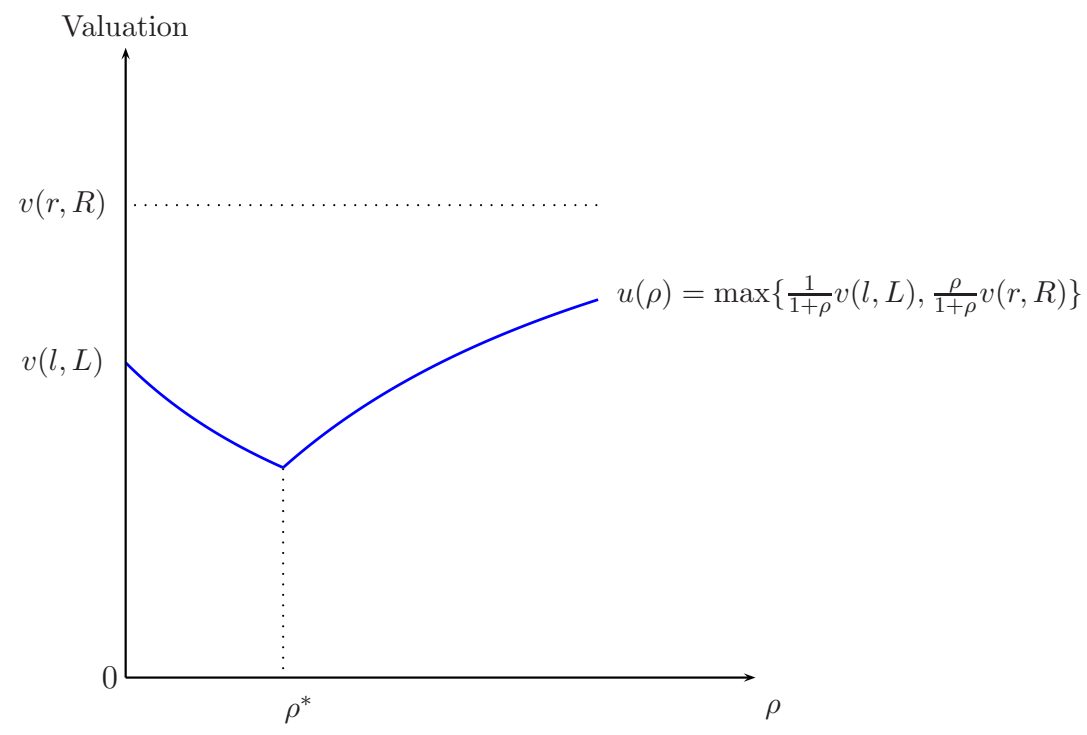

Figure 1: Continuation value as a function of the likelihood ratio of the bidder who wins a unit in the auction, before he makes the action choice. Assumption 1 implies that $u(\rho)$ is a nonmonotonic function which is minimized at $\rho^{*}$ as depicted here.

suppose that $\rho(s)$ is continuous and is strictly increasing in s. Note that $\rho(0)>\rho^{*}$ implies that $\rho(s)>\rho^{*}$ for all $s \in[0,1]$. This is a consequence of Assumption 3. If $\rho(0)>\rho^{*}$, then $u(\rho(s))$ is an increasing function of $s$, the bidder with signal zero has the lowest expected valuation for the good, and the bidder with signal one has the highest expected valuation for the good. See figure 2 for a depiction.

Now suppose that new information arrives in the form of an additional public signal $s^{*} \in$ $\{d, u\}$ which is conditionally independent from the bidders' private signals. After a bidder observes the additional public signal $s^{*}$, his likelihood ratio is given by $\rho\left(s, s^{*}\right)=\rho(s) \frac{\operatorname{Pr}\left(s^{*} \mid R\right)}{\operatorname{Pr}\left(s^{*} \mid L\right)}$, where $\operatorname{Pr}\left(s^{*} \mid \omega\right)$ denotes the probability of observing signal $s^{*}$ given that the state is $\omega \in$ $\{L, R\}$. If the realization of the public signal is $s^{*}=d$, then a sufficiently strong public signal can completely reverse the ordering of the bidders. ${ }^{10}$ In other words, it is possible that the value function $u(\rho(s, d))$ is decreasing in $s$ even though the value function $u(\rho(s))$ was increasing in s before the bidders observed the public signald. See figure 3 (a) for a depiction. Similarly, it is also possible for the function $u(\rho(s, d))$ to be non monotonic even though the value function was monotonic before the bidders observed the public signal d. See figure 3 (b) for a depiction.

\footnotetext{
${ }^{10}$ For example, if $\frac{\operatorname{Pr}(d \mid R)}{\operatorname{Pr}(d \mid L)}<\rho^{*} / \rho(1)$ or, equivalently, if $\rho(1, d)<\rho^{*}$, then $\rho(s, d)<\rho^{*}$ for all $s \in[0,1]$. This is because $\rho(s) \leq \rho(1)$ for all $s \in[0,1]$. However, $\rho(s, d)<\rho^{*}$ for all $s \in[0,1]$ implies that the value function $u(\rho(s, d))$ is decreasing in $s$ even though the value function $u(\rho(s))$ was increasing in $s$ before the bidders observed the public signal $d$.
} 


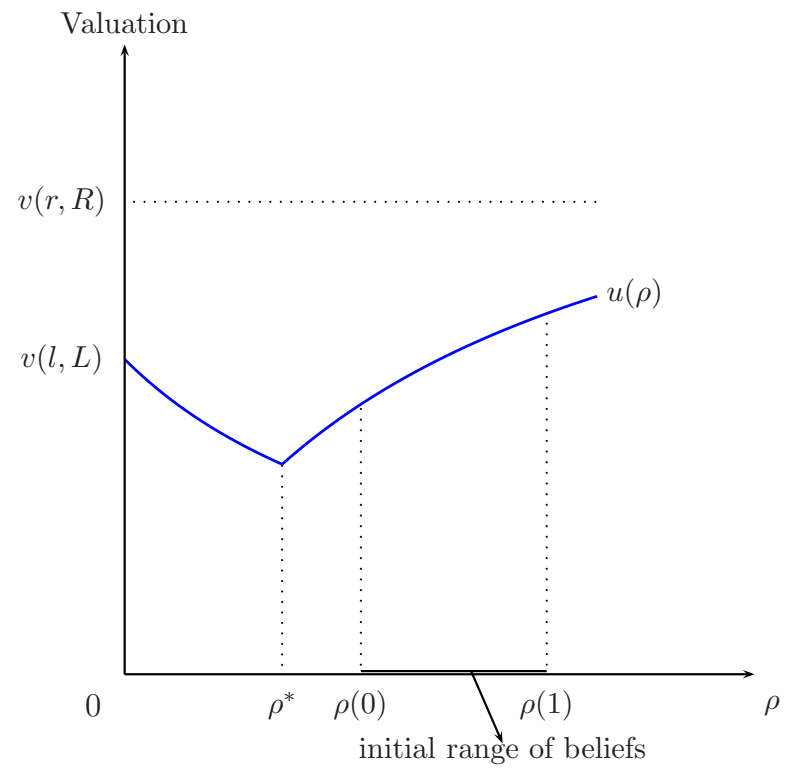

Figure 2: This figure shows the initial range of beliefs, expressed as likelihood ratios, on the beliefvalue graph. Note that the types that assign a higher probability to state $R$ (i.e., types with higher signals) are the types with higher values because $\rho(0) \geq \rho^{*}$.

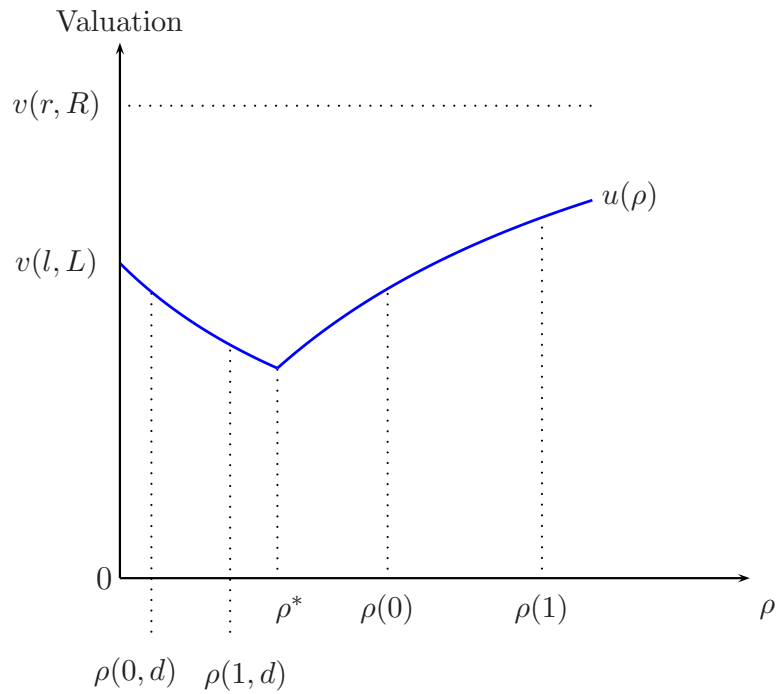

(a)

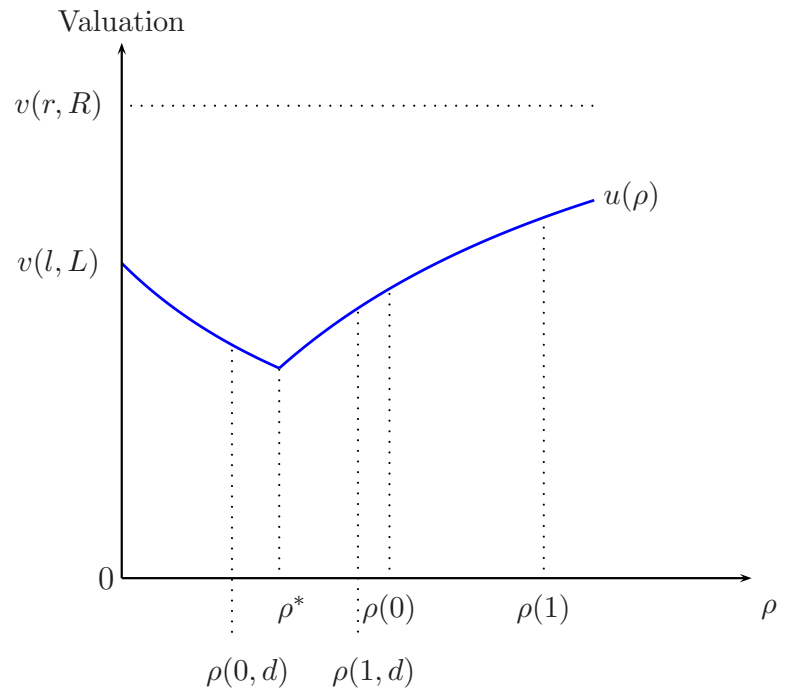

(b)

Figure 3: This figure shows both the range of initial beliefs and two examples of the range of beliefs that bidders could hold if they received an extra signal $d$ which suggests that the state is $L$. The signal $d$ that the bidders receive is more strongly in favor of $L$ in $(a)$ than in $(b)$. 
REMARK 3 In auctions where there is no ex-post action, the ordering of the bidders according to their valuation cannot change as a result of new information. ${ }^{11}$ To demonstrate this point, we continue with the example in Remark 1. An auction in which there is no ex-post action can be modeled in our framework by restricting the actions available to the bidders to only one action, e.g., restricting $A=\{r\}$. In this case, $u(\rho)$ is an increasing function of $\rho$ for all $\rho$. Consequently, both $u(\rho(s))$ and $u\left(\rho\left(s, s^{*}\right)\right)$ are increasing functions of $s$, for every $s^{*} \in\{u, d\}$.

2.3. Strategies and equilibrium Each bidder submits a bid after observing his signal. A bidding strategy for player $i$ is a measure $H_{i}$ on $[0,1] \times[0, \infty)$ with marginal distribution $F(s)=\pi F(s \mid R)+(1-\pi) F(s \mid L)$ on its first coordinate (see ?). The set of all bidding strategies is $\Sigma$. A strategy is pure if there is a function $b:[0,1] \rightarrow[0, \infty)$ such that $H\left(\{s, b(s)\}_{s \in[0,1]}\right)=$ $1 .{ }^{12}$

Each winner chooses an action from the set of actions $A$. Hence, the action strategy is a mapping from a bidder's signal, his bid, and the winning price to an action, $a_{i}: S \times$ $[0, \infty) \times[0, \infty) \rightarrow A$. Since the bidders' actions do not affect other bidders' payoffs, confining attention to pure strategy actions is without loss of generality.

Every bidding strategy profile $H:=\left\{H_{i}\right\}_{i \in\{1,2, \ldots, n\}}$, together with nature's choice of state and signals, induces a joint probability distribution $\bar{H}$ over signals, prices, and winners. Let $\rho_{i}(s, b, p$, win $)$ denote the posterior likelihood of state $R$ for a bidder $i$ who receives a signal $s$, bids $b$, and wins an object from the auction at price $p \leq b$. Such a bidder's optimal action choice is $r$ if $\rho_{i}(s, b, p$, win $)>\rho^{*}$, and $l$ if $\rho_{i}(s, b, p$, win $)<\rho^{*}$. Moreover, the continuation payoff of such a bidder is $u\left(\rho_{i}(s, b, p\right.$, win $\left.)\right)$. The payoff to bidder $i$ of the bidding strategy $H_{i}$ when the bidders other than $i$ are following the strategy profile $H_{-i}$ is given by:

$$
U_{i}\left(H_{i}, H_{-i}\right)=\int_{s \in S} \int_{b \in[0, \infty)} \int_{p \in[0, \infty)} u\left(\rho_{i}(s, b, p, \text { win })\right) d \bar{H}(s, b, p, i \text { wins a unit }) .
$$

A bidding strategy profile $H$ is a Nash equilibrium if

$$
U_{i}\left(H_{i}, H_{-i}\right) \geq U_{i}\left(H^{\prime}, H_{-i}\right) \text { for every } H^{\prime} \in \Sigma \text { and } i \in\{1,2, \ldots, n\} \text {. }
$$

In the rest of the paper, we will construct equilibria in which bidders use pure bidding strategies. We will also restrict our attention to pure symmetric Nash equilibria, which are equilibria where each bidder uses the same pure bidding strategy, i.e., $b_{i}=b_{j}$ for every two bidders $i$ and $j$. The term $\operatorname{Pr}_{b}$ denotes the probability distribution induced by the pure and

\footnotetext{
${ }^{11}$ See, for example, ? or ?.

${ }^{12}$ Of course, if $b$ represents $H$, then so will any function that agrees with $b$ for almost every $s \in[0,1]$.
} 
symmetric bidding strategy profile where each bidder uses the bidding strategy $b$ over states of the world, signal and bid distributions, allocations, and prices.

\section{Large Markets and the Failure of Information Aggregation}

In this section we present our main result as Theorem 1. In Theorem 1, we constuct a sequence of equilibria for auctions $\left\{\Gamma_{n}\right\}_{n=1}^{\infty}$ where the $n^{\text {th }}$ auction $\Gamma_{n}$ has $n$ bidders and $\lfloor\kappa n\rfloor$ objects for sale. ${ }^{13}$ In the remainder of the paper, we will proceed as if $\kappa n$ is an integer for expositional simplicity. We assume that the other parameters of the auctions, i.e., $(v, F, \pi, \kappa)$, are constant along the sequence and satisfy all the assumptions that we have already made. For the sequence of equilibria we construct, equilibrium price reveals no information about the state of the world at the limit where there is an arbitrarily large number of bidders. Although the limit equilibrium price reveals no information, bidders do learn some information about the state of the world through rationing. However, the amount of information that they learn is limited, and incorrect ex-post actions are played frequently.

3.1. Information aggregation. Here we formally define information aggregation and its failure. Our object of study is a sequence of bidding functions $\boldsymbol{b}=\left\{b_{n}\right\}_{n=m}^{\infty}$. We say that the sequence $\boldsymbol{b}$ is an equilibrium sequence if $b_{n}$ is part of a symmetric Nash equilibrium of $\Gamma_{n}$ for each $n$.

Suppose that the number of bidders $n$ is large. In this case, the law of large numbers implies that observing the signals $\left(s_{1}, \ldots, s_{n}\right)$ conveys precise information about the state of the world $\omega \in\{L, R\}$. The bidding function $b_{n}$ determines a price $p^{*}$ for the auction $\Gamma_{n}$ given any realization of signals $\left(s_{1}, \ldots, s_{n}\right)$. We say that information is aggregated in the auction if this price $p^{*}$ also conveys precise information about the state of the world. More precisely, $(i)$ if the likelihood ratio $\frac{\operatorname{Pr}_{b_{n}}\left(p^{*} \mid R\right)}{\operatorname{Pr}_{b_{n}}\left(p^{*} \mid L\right)}$ is close to zero (i.e., if it is arbitrarily more probable that we observe such a price when $\omega=L$ ), then an outsider who observes price $p^{*}$ learns that the state is $L$. Alternatively, $(i i)$ if the likelihood ratio $\frac{\operatorname{Pr}_{b_{n}}\left(p^{*} \mid R\right)}{\operatorname{Pr}_{b_{n}}\left(p^{*} \mid L\right)}$ is arbitrarily large, then an outsider who observes price $p^{*}$ learns that the state is $R$. If the probability that we observe a price that satisfies either $(i)$ or $(i i)$ is arbitrarily close to one, then we say that the equilibrium sequence $\boldsymbol{b}$ fully aggregates information. Conversely, if the likelihood ratio $\frac{\operatorname{Pr}_{b_{n}}\left(p^{*} \mid R\right)}{\operatorname{Pr}_{b_{n}}\left(p^{*} \mid L\right)}$ is close to one, i.e., if we are equally likely to observe price $p^{*}$ in either of the two states, then an outsider who observes price $p^{*}$ learns arbitrarily little information about the state of the world. If the probability that we observe such a price is arbitrarily close to one, then we say that the equilibrium sequence $\boldsymbol{b}$ aggregates no information. The precise definitions are as follows:

\footnotetext{
13 The term $\lfloor\kappa n\rfloor$ refers to the highest integer not bigger than $\kappa n$.
} 
DEFINITION 1 An equilibrium sequence b aggregates no information if, for any $\epsilon>0$,

$$
\lim _{n \rightarrow \infty} \operatorname{Pr}_{b_{n}}\left(p_{n} \in\left\{p \in[0, \infty): \frac{\operatorname{Pr}_{b_{n}}(p \mid R)}{\operatorname{Pr}_{b_{n}}(p \mid L)} \in(1-\epsilon, 1+\epsilon)\right\}\right)=1 .
$$

An equilibrium sequence b fully aggregates information if, for any $\epsilon>0$,

$$
\left.\lim _{n \rightarrow \infty} \operatorname{Pr}_{b_{n}}\left(p_{n} \in\left\{p \in[0, \infty): \frac{\operatorname{Pr}_{b_{n}}(p \mid R)}{\operatorname{Pr}_{b_{n}}(p \mid L)} \in[0, \epsilon) \cup(1 / \epsilon, \infty)\right)\right\}\right)=1
$$

REMARK 4 Our definition of information aggregation differs from the definition provided by ?. In their model, the state of the world is defined as the value of the object and each bidder receives a signal about that value. Therefore, they say that information is aggregated if the equilibrium prices converge to the true value of the object (i.e., if the price converges to the state of the world) as the market grows large. In their setup, each state represents a distinct value for the object, so when information is aggregated in their model with their definition, then it is also aggregated under our definition. Therefore, if information aggregation fails using our definition, then it will also fail under the definition of?.

3.2. Failure of information aggregation. Our main theorem shows that if, in addition to Assumptions 1-4, two conditions are satisfied, then there exists an equilibrium sequence b which aggregates no information. The first condition that we require for the theorem is as follows:

Condition $1 \quad \rho(0)>\rho^{*}$.

If this condition is satisfied, then all the bidders would choose action $r$ if they acted solely on the information contained in their private signal. See figure 2 for a depiction of a situation that satisfies Condition 1.

Recall that $s_{R}^{\kappa} \in S$ is the signal such that $F\left(s_{R}^{\kappa} \mid R\right)=1-\kappa$. The second condition we require for the theorem is as follows:

Condition $2 \inf _{s>s_{R}^{\kappa}} u(\rho(s))<v(l, L)$.

Under this condition, if a bidder who received a signal arbitrarily close to $s_{R}^{\kappa}$ chooses an action based solely on this signal, then this bidder's expected valuation is lower than $v(l, L)$. Therefore, a sufficiently strong additional signal in favor of state $L$ can increase the expected valuation of such a bidder. See figure 4 for a depiction of a situation where both Conditions 1 and 2 are satisfied.

Our main theorem is as follows: 


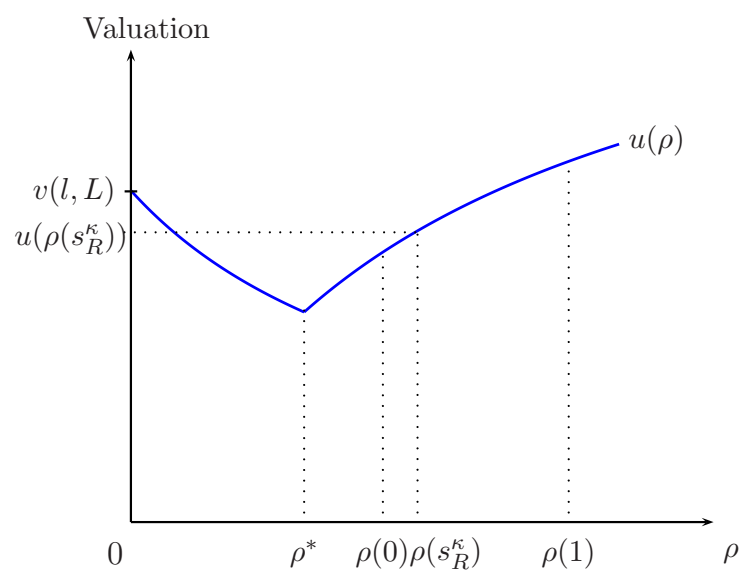

Figure 4: This figure shows the initial range of beliefs, expressed as likelihood ratios, on the beliefvalue graph. Note that the bidders who assign higher probability to state $R$, i.e., the bidders with higher signals, are the bidders with higher value.

Theorem 1 Suppose that Assumptions 1-4 hold. If Condition 1 and Condition 2 also hold, then there exists an equilibrium sequence $\boldsymbol{b}$ which reveals no information.

Proof: see Appendix.

We prove this theorem by constructing an equilibrium sequence which aggregates no information. In this construction, each bidding function $b_{n}$ in the equilibrium sequence $\boldsymbol{b}$ is a nondecreasing function of $s$. In this construction, Condition 1 allows us to construct an equilibrium sequence in which each bidding function $b_{n}$ is nondecreasing in $s$. Condition 2 , on the other hand, allows us to ensure that the equilibrium sequence that we construct aggregates no information about the state of the world.

3.3. Sketch of the construction. In this section, we sketch the ideas behind constructing the equilibrium sequence $\mathbf{b}$ whose existence Theorem 1 claims. Specifically, we construct an equilibrium in which no information is aggregated in a hypothetical market with a continuum of bidders with mass one and a continuum of objects with mass $\kappa$. Focusing on a hypothetical market with a continuum of bidders allows us to capture the main properties of the equilibrium sequence $\mathbf{b}$ for a market with a finite but large number of bidders while allowing us to avoid the more technical details involved in describing such equilibria for finite markets. In section 5.2, we discuss in detail how one can use the intuition developed here to construct the equilibrium sequence $\mathbf{b}$ for a sequence of finite markets. In order to simplify the exposition, we also assume that $\rho(s)$ is a continuous, strictly increasing function of the signal $s$. Also, in what follows we repeatedly use the fact that the value function $u(\cdot)$ is strictly decreasing in the interval $\left[0, \rho^{*}\right]$ and strictly increasing in the interval $\left[\rho^{*}, \infty\right)$. 


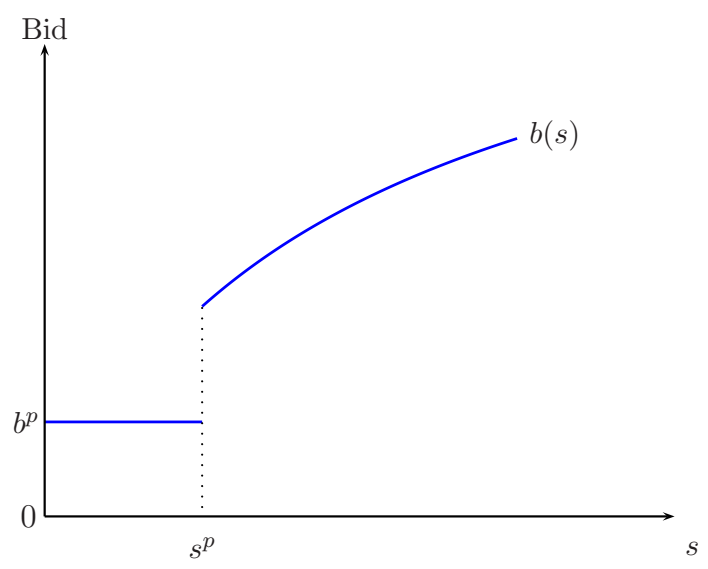

Figure 5: A typical equilibrium bid function that is monotonic and increasing. Buyers with signals below a cutoff $s^{p}$ bid a pooling bid $b^{p}$, and those with signals above $s^{p}$ bid according to the bid function in ?, i.e., $b(s)=\rho\left(s_{1}=s, Y_{n-1}^{k}=s\right)$ for $s>s^{p}$.

We construct an equilibrium in which the equilibrium bidding function $b$ is constant on the interval $\left[0, s^{p}\right)$ for some cutoff signal $s^{p}>s_{R}^{\kappa}$, which we calculate further below (i.e., $b(s)=b^{p}$ for all $\left.s \in\left[0, s^{p}\right)\right)$, and the bidding function is strictly increasing on the interval $\left(s^{p}, 1\right]$. We call the bid $b^{p}$ (i.e., the bid submitted by all bidders with signals in the interval $\left.\left[0, s^{p}\right)\right)$ the pooling bid. See figure 5 for a depiction of the bidding function $b$.

In this equilibrium, the following properties hold true:

( $i$ ) The auction price is equal to the pooling bid in either state of the world, and hence conveys no additional information about the state of the world. The auction price is always equal to the bidding price because $s^{p}$ exceeds $s_{R}^{\kappa}$. The fact that $s^{p}$ exceeds $s_{R}^{\kappa}$ implies that for any price $p^{\prime}>b^{p}$, the mass of bidders who submit a bid greater than or equal to $p^{\prime}$ is strictly less than the mass of objects available, i.e., the measure of the set $\left\{s: b(s) \geq p^{\prime}\right\}$ is strictly less than $\kappa$ in both states.

(ii) Bidders with signals that exceed $s^{p}$, i.e., those bidders whose bid exceeds the pooling price, are always allocated an object and always choose action $r$. These bidders choose action $r$ because they obtain no new information from the auction price and because choosing $r$ is optimal based solely on their private signal.

(iii) Bidders with signals less than $s^{p}$ who are allocated an object, i.e., the bidders who bid the pooling price, take action $l$. Although the price conveys no information about the state, the fact that a bidder wins an object by bidding the pooling price is a strong signal favoring state $L$ which induces that bidder to choose action $l$. Winning an object by bidding the pooling price is a strong signal favoring state $L$ because the mass of bidders bidding the pooling price exceeds the mass of objects to be allocated to bidders who bid the pooling price. Moreover, a bidder is more likely to be allocated a good in 
state $L$ than in state $R$. We discuss this issue in more detail below.

We now discuss how to calculate the cutoff signal $s^{p}$. The cutoff signal $s^{p}$ is the signal which leaves a bidder indifferent between bidding the pooling bid and bidding slightly above the pooling bid. As a first step in calculating $s^{p}$, we calculate a bidder's payoff if he bids slightly above the pooling bid, and if he bids the pooling bid and wins an object.

Payoff from bidding slightly above the pooling bid. If a bidder bids above the pooling bid, then she wins an object with certainty. The posterior belief of a bidder who wins an object by bidding above the pooling bid is equal to her initial beliefs. This is because the auction price is always equal to the pooling bid in this equilibrium and conveys no information. Consequently, the expected value of the object to a bidder with signal $s$ if she bids above the pooling bid is $u(\rho(s))$.

Payoff from bidding the pooling bid. We now calculate the value of the object for a bidder who receives the cutoff signal $s^{p}$ if he bids the pooling bid and wins a unit, when $s^{p} \geq s_{R}^{\kappa}$. In such an event, this bidder has an extra piece of information, which comes from the fact that he wins a unit while bidding the pooling bid. In particular, a fraction $1-F\left(s^{p} \mid \omega\right)$ of bidders bid strictly above the pooling bid and each wins an object with certainty regardless of the state. The fraction of objects that remains to be delivered to bidders who choose the pooling bid is $\kappa-\left(1-F\left(s^{p} \mid \omega\right)\right)$. Since the number of objects remaining to be delivered is less than the number of bidders, there is rationing among the bidders at the pooling bid. Consequently, the belief of type $s^{p}$ (represented as the likelihood ratio) if he bids the pooling bid and wins the object is as follows:

$$
\rho^{p}\left(s^{p}\right):=\rho\left(s^{p}\right) \frac{\kappa-\left(1-F\left(s^{p} \mid R\right)\right)}{F\left(s^{p} \mid R\right)} / \frac{\kappa-\left(1-F\left(s^{p} \mid L\right)\right)}{F\left(s^{p} \mid L\right)}=\rho\left(s^{p}\right) \frac{\kappa-\left(1-F\left(s^{p} \mid R\right)\right)}{\kappa-\left(1-F\left(s^{p} \mid L\right)\right)} \frac{F\left(s^{p} \mid L\right)}{F\left(s^{p} \mid R\right)}
$$

where the ratio $\Delta\left(s^{p}\right):=\frac{\kappa-\left(1-F\left(s^{p} \mid R\right)\right)}{F\left(s^{p} \mid R\right)} / \frac{\kappa-\left(1-F\left(s^{p} \mid L\right)\right)}{F\left(s^{p} \mid L\right)}$ reflects the extra information that a bidder learns from winning an object at the pooling bid. If a bidder with signal $s^{p}$ bids the pooling bid and wins the object, then the expected value of the object to him is equal to $u\left(\rho^{p}\left(s^{p}\right)\right)$.

REMARK 5 It is straightforward to verify that $\Delta\left(s^{p}\right)<1$, that is, winning an object at the pooling bid is more likely in state $L$ than in state $R$; winning an object at the pooling bid is therefore an additional signal in favor of state L. In the context of the auction models of? or ?, the fact that $\Delta\left(s^{p}\right)<1$ is commonly referred to as the loser's curse. ${ }^{14}$ Intuitively, the loser's curse holds because if the state is L, then the weak MLRP (Assumption 2) implies

\footnotetext{
${ }^{14}$ The loser's curse is defined in the setting with finitely many bidders; however, the idea extends naturally to the hypothetical setting with a continuum of bidders.
} 


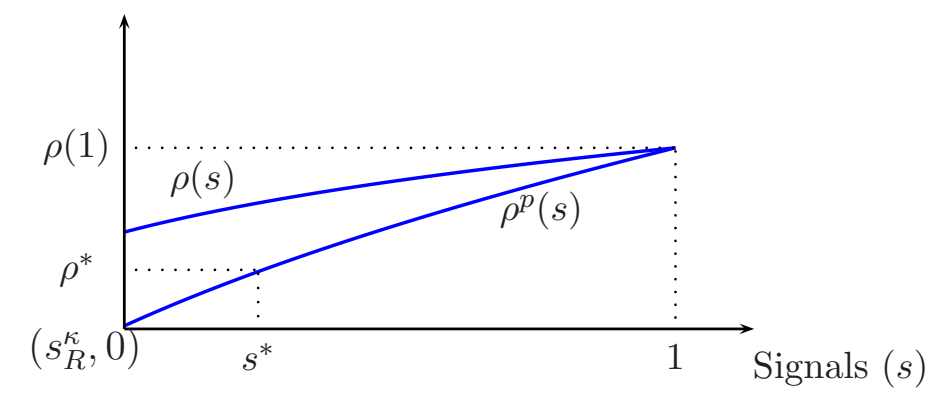

Figure 6: This figure depicts the functions $\rho$ and $\rho^{p}$, in the range $\left[s_{R}^{\kappa}, 1\right]$. Notice that $\rho(s) \geq \rho^{p}(s)$; $\rho^{p}\left(s_{R}^{\kappa}\right)=0 ;$ and $\rho(1)=\rho^{p}(1)$.

that fewer bidders choose a bid which exceeds the pooling bid, and therefore more goods are left over to be allocated to the bidders who choose the pooling bid. We discuss the loser's curse in greater detail in Section 3.5 .

As we stated above, the cutoff signal $s^{p}$ is the signal which leaves a bidder indifferent between bidding the pooling bid and bidding slightly above the pooling bid. In other words, the cutoff signal is defined implicitly by the following equation:

$$
u\left(\rho\left(s^{p}\right)\right)=u\left(\rho^{p}\left(s^{p}\right)\right)
$$

We now argue that this cutoff signal is unique. Specifically, we show that there is a unique signal $s>s_{R}^{\kappa}$ such that $u(\rho(s))=u\left(\rho^{p}(s)\right)$, and we denote this signal by $s^{p}$. Note that $\rho^{p}\left(s_{R}^{\kappa}\right)=0$ and $\left.\lim _{s}\right\rfloor_{R}^{\kappa} \rho^{p}(s)=\rho^{p}\left(s_{R}^{\kappa}\right)$. By Condition 2, we have $v(l, L)>u\left(\rho\left(s_{R}^{\kappa}\right)\right)$, and hence, $u\left(\rho^{p}\left(s_{R}^{\kappa}\right)\right)=u(0)=v(l, L)>u\left(\rho\left(s_{R}^{\kappa}\right)\right.$. Let $s^{*}$ denote the unique signal such that $\rho^{p}\left(s^{*}\right)=\rho^{*}$, and note that $s^{*} \in\left(s_{R}^{\kappa}, 1\right)$. The function $u(\rho(s))$ is strictly increasing in $s$, and $u(\rho(s))>u\left(\rho^{*}\right)$ for all $s$. The function $u(\rho(s))$ is strictly increasing because $\rho(s)>\rho^{*}$ (by assumption), because $\rho(s)$ is strictly increasing in $s$, and because $u(\rho)$ is strictly increasing for any $\rho \in\left(\rho^{*}, \rho\right]$. Also, the function $u\left(\rho^{p}(s)\right)$ is strictly decreasing in $s$ for all $s \in\left[s_{R}^{\kappa}, s^{*}\right]$, is strictly increasing in $s$ for all $s \in\left[s^{*}, 1\right]$, and reaches its minimum at $u\left(\rho^{p}\left(s^{*}\right)\right)=u\left(\rho^{*}\right)$. Consequently, the two functions must cross at some point $s^{p} \in\left(s_{R}^{\kappa}, s^{*}\right)$. There is a unique such point, $s^{p}$, because $\rho^{p}(s)=\rho(s) \Delta(s)<\rho(s)$ for all $s \in\left[s_{R}^{\kappa}, 1\right)$. See figures 6 and 7 for depictions.

We now check that bidders will not want to deviate from the equilibrium we described. We first argue that bidders with signals lower than $s^{p}$ cannot profitably deviate from the equilibrium by choosing a bid that exceeds the pooling bid. If a bidder with signal $s<s^{p}$ 


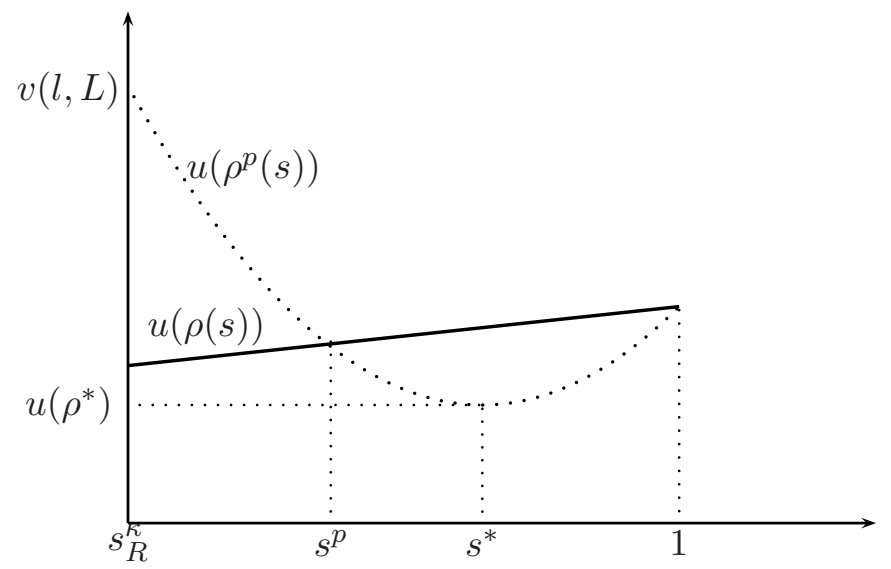

Figure 7: This figure depicts the value of the object to the cutoff type as a function of the choice of the cutoff type.

deviates and bids above the pooling bid, then she wins an object with certainty and pays the pooling bid $b^{p}=u\left(\rho\left(s^{p}\right)\right)$. In this case, her posterior and prior likelihood ratios coincide and are equal to $\rho(s)$. However, Condition 1 implies that $\rho^{*}<\rho(s)<\rho\left(s^{p}\right)$, and therefore we have $u(\rho(s))<b^{p}=u\left(\rho\left(s^{p}\right)\right)$, i.e., the auction price exceeds the expected valuation, conditional on winning, of the bidder with signal $s$. See figure 8 for a depiction of this argument for the case of $s=0$.

We now argue that a bidder with signal $s>s^{p}$ cannot profitably deviate from equilibrium by choosing the pooling bid. If the bidder sticks to the equilibrium strategy, then she wins an object with certainty and her payoff is equal to $u(\rho(s))-b^{p}$, a payoff which is strictly positive. If she deviates instead and chooses the pooling bid, then, conditional on winning, her posterior is equal to $\rho^{*}(s):=\rho(s) \Delta\left(s^{p}\right)$. Note that $\rho^{p}\left(s^{p}\right)<\rho^{*}(s)<\rho(s)$. To see that this deviation is not profitable, consider two cases. First, if $\rho^{*}(s) \geq \rho^{*}$, then $u\left(\rho^{*}(s)<\right.$ $u(\rho(s))$. This is because $\rho^{*}(s)=\rho(s) \Delta\left(s^{p}\right)<\rho(s)$ and because $u(\cdot)$ is increasing on $\left[\rho^{*}, \infty\right)$. Alternatively, if $\rho^{*}(s) \leq \rho^{*}$, then $u\left(\rho^{*}(s)\right)-b^{p}=u\left(\rho(s) \Delta\left(s^{p}\right)\right)-u\left(\rho^{p}\left(s^{p}\right)\right)<0$. This is because $\rho^{*}(s)=\rho(s) \Delta\left(s^{p}\right)>\rho\left(s^{p}\right)$ and because $u(\cdot)$ is decreasing on $\left[0, \rho^{*}\right]$. See figure 8 for a depiction.

3.4. Properties of equilibrium. There are a number of novel properties of the equilibrium that we constructed for Theorem 1. In particular, the properties listed below are satisfied as the number of bidders grow arbitrarily large. Note that none of these properties is present in a standard auction where there are no ex-post actions.

(i) No information aggregation: the equilibrium price aggregates no information even when the market grows arbitrarily large. We have already discussed the intuition behind this property in the preceding section.

(ii) Learning from one's own bid: The posterior beliefs of the bidders who win an object depend on their bid. In particular, the posterior beliefs of the bidders who win 


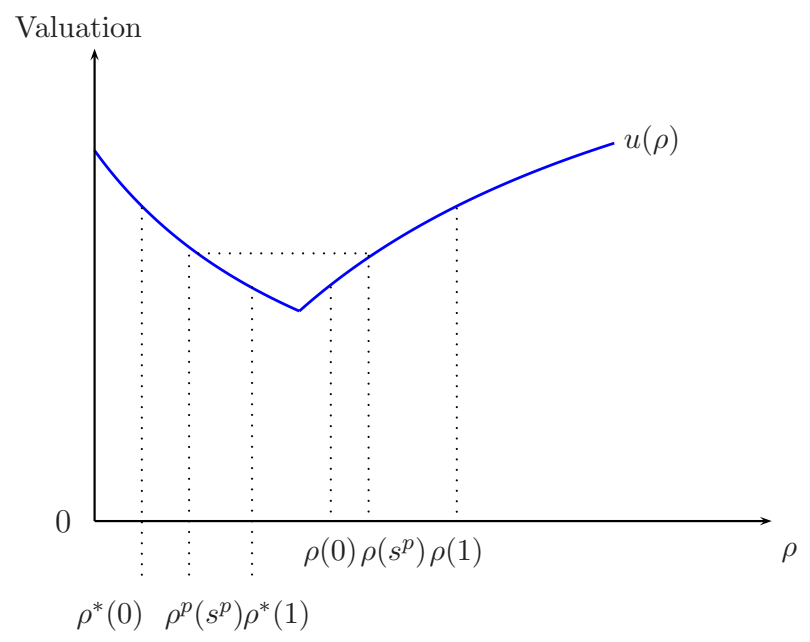

Figure 8: This figure depicts the posterior beliefs of bidders with signals $0, s^{p}$, and 1 under two cases: $(i)$ If they bid the pooling bid and win the object. In this case, their posterior likelihood ratios are $\rho^{*}(0)=\rho(0) \Delta\left(s^{p}\right), \rho^{p}\left(s^{p}\right)=\rho\left(s^{p}\right) \Delta\left(s^{p}\right)$, and $\rho^{*}(1)=\rho(1) \Delta\left(s^{p}\right)$. (ii) if they bid above the pooling bid and win a unit at the pooling price. In this case, the bidders obtain no new information and therefore their posterior and prior likelihood ratios coincide, i.e., their posterior likelihood ratios are also equal to $\rho(0), \rho\left(s^{p}\right)$, and $\rho(1)$. Bidders with signal 0 strictly prefer to bid the pooling bid, those with signal $s^{p}$ are indifferent between bidding the pooling bid and above it, and those with signal 1 strictly prefer to bid above the pooling bid. Note that the pooling bid satisfies the equality $b^{p}=u\left(\rho\left(s^{p}\right)\right)=u\left(\rho^{p}\left(s^{p}\right)\right)$. 
an object by bidding above the pooling bid is equal to their prior belief. This is because the auction price reveals no new information. In contrast, the posterior likelihood ratio of bidders who win an object by bidding the pooling bid is equal to their prior belief, $\rho(s)$, multiplied by the constant $\Delta\left(s^{p}\right) \in(0,1)$. Hence, a bidder's bid may affect the information he will have if he wins a unit.

(iii) Incorrect choice of action: Because prices convey no new information and because bidders' posterior beliefs are heterogeneous, a strictly positive fraction of bidders take the wrong action in equilibrium. Therefore, inefficiency persists even in a large market in which the outcome would have been efficient if one could use all of the signals observed by the bidders. In particular, irrespective of the state of the world, all bidders who win an object at the pooling bid choose action $l$ and all other bidders who win an object choose action $r$. Consequently, the proportion of bidders choosing the wrong action is equal to $1-F\left(s^{p} \mid L\right)$ and $\kappa-\left(1-F\left(s^{p} \mid R\right)\right)$ when the state of the world is $L$ and $R$, respectively. Note that the total expected surplus in the equilibrium that we construct is equal to this expression:

$$
\pi v(r, R)\left(1-F\left(s^{p} \mid R\right)\right)+(1-\pi) v(l, L)\left(\kappa-\left(1-F\left(s^{p} \mid L\right)\right)\right)
$$

Because $\rho(0)>\pi^{*}$ (by assumption), the equilibrium surplus is strictly decreasing in $s^{p}$.

(iv) Positive profits: The expected profit of each bidder, except the bidder who receives signal $s^{p}$, is strictly positive in equilibrium. Even though the equilibrium price is equal to the pooling bid, the bidders who submit the pooling bid also make positive profits. In particular, the following profit function is obtained:

$$
\Pi(s):= \begin{cases}\frac{\kappa-\left(1-F\left(s^{p} \mid L\right)\right)}{F\left(s^{p} \mid L\right)(1+\rho(s))}\left(v(l, L)-b^{p}\left(1+\rho(s) \Delta\left(s^{p}\right)\right)\right) & \text { if } s \leq s^{p} \\ \frac{\rho(s)}{1+\rho(s)} v(r, R)-b^{p} & \text { if } s \geq s^{p}\end{cases}
$$

Note that $\Pi\left(s^{p}\right)=0$ by construction, $\Pi(s)>0$ for all $s<s^{p}$ and for all $s>s^{p}$.

(v) Valuable information: The value of information, i.e., the value of receiving a signal, is strictly positive for the bidders. This is because winning an object at the pooling bid is only partially informative and the equilibrium price is uninformative, while, on the other hand, signals provide partial information about the state as a consequence of Assumption 5 .

3.5. Loser's curse. A novel feature of our equilibrium construction is the existence of a pooling bid $b^{p}$, which is chosen by bidders who receive a signal in the interval $\left[0, s^{p}\right]$. 
In other words, in the equilibrium that we construct, there is an atom in the equilibrium bid distribution at $b^{p}$. In sharp contrast, the existence of a pooling bid, i.e., an atom in the equilibrium bid distribution, is not possible in the symmetric equilibria of the auction models of ? or ?, where there is no ex-post action, even when the signal space is discrete. The existence of a pooling bid in our model and the impossibility of pooling in auctions without ex-post actions are both consequences of the loser's curse, i.e., the fact that the probability of winning an object at the pooling bid in state $L$ is strictly higher than the probability of winning an object at the pooling bid in state $R$. Equivalently, a bidder is more convinced that the state is $\mathrm{R}$ when he does not win an object than when he does, provided that the price is the pooling bid and he bid the pooling bid.

In the previous section, we discussed the role of the loser's curse in sustaining our equilibrium construction. We now discuss how the loser's curse precludes a pooling bid in an auction in which there is no ex-post action. Such an auction can be modeled in our framework by restricting the actions available to the bidders to only one action, for example, taking $A$ to equal $\{r\}$. In this case, $u(\rho)$ is an increasing function of $\rho$ and so $u(\rho(s))$ is also an increasing function of $s$. Consider again the equilibrium discussed in the previous section. In that construction, the bidding function is weakly increasing in the signals, i.e., $b(s) \geq b\left(s^{\prime}\right)$ whenever $s \geq s^{\prime}$. Moreover, all bidders with a signal $s<s^{p}$ choose the pooling bid $b^{p}$. We now argue that such an equilibrium is not possible because of the loser's curse. If a bidder with signal $s<s^{p}$ chooses the pooling bid, then, conditional on winning an object, her payoff is equal to $u\left(\rho(s) \Delta\left(s^{p}\right)\right)-b^{p}$. If she deviates instead and bids above the pooling bid, then she wins an object with certainty and pays the pooling bid $b^{p}$. In this case, her posterior is equal to $\rho(s)$ and consequently her payoff, if she deviates, is equal to $u(\rho(s))-b^{p}$. However, the loser's curse, i.e., $\Delta\left(s^{p}\right)<1$, implies that $\rho(s) \Delta\left(s^{p}\right)<\rho(s)$ and hence, that $u\left(\rho(s) \Delta\left(s^{p}\right)\right)<u(\rho(s))$. Therefore, this is a profitable deviation as long as $u\left(\rho(s) \Delta\left(s^{p}\right)\right) \geq b^{p}$, which shows that this cannot be an equilibrium.

Intuitively, not winning an object at the pooling bid, when the auction price is equal to the pooling bid, is a strong signal in favor of state $R$. Therefore, whenever the auction price is equal to the pooling price, a bidder would rather increase his bid slightly and ensure that he wins an object. Following this intuition, ? show that, if there is no ex-post action, there is no symmetric equilibrium where the bid distribution includes an atom (or pooling).

\section{Information Aggregation Failures in Monotone Equilibria}

In the previous section, while discussing Theorem 1, we described equilibria in which no information is aggregated by the price. A prominent property of the equilibrium we described is that equilibrium bids are nondecreasing in the signal that a bidder receives, i.e., the bidding 
function is a nondecreasing function of signals. In this section, in order to demonstrate the robustness of Theorem 1, we characterize all symmetric equilibria in which the bid function is a monotonic function of signals (Lemma 1). We then use our characterization to show that information cannot be fully aggregated in equilibria in which the bidding function is monotonic (Theorem 2). Moreover, we show that equilibria in which the bidding function is monotonic exist under a mildly restrictive condition (Theorem 2). Consequently, our results in this section show that $i$ the failure of information aggregation is inherent in equilibria in which the bidding function is monotonic; and moreover, ii such equilibria exist for a wide range of parameter values.

Recall that our object of study is a sequence of equilibrium bidding functions $\boldsymbol{b}=\left\{b_{n}\right\}_{n=m}^{\infty}$. We say that a bidding function is nondecreasing (nonincreasing) if $b(s)$ is a nondecreasing (nonincreasing) function of $s$, and we say that a bidding function is monotone if it is either nondecreasing or nonincreasing. We say that an equilibrium sequence $\boldsymbol{b}$ is nondecreasing (nonincreasing) if $b_{n}$ is part of a symmetric Nash equilibrium of $\Gamma_{n}$ and if $b_{n}$ is a nondecreasing (nonincreasing) bidding function for each $n$. We say that a sequence $\boldsymbol{b}$ is monotonic if the sequence is either nondecreasing or nonincreasing. We begin by characterizing nondecreasing equilibrium bidding functions. ${ }^{15}$

LEMma 1 (Characterization) Suppose that Assumptions 1-4 hold. Every equilibrium bidding function $b$ that is nondecreasing satisfies the following conditions:

(i) There is a cutoff signal $s^{p} \in[0,1]$ and a pooling bid $b^{p}$ such that $b(s)=b^{p}$ for every $s<s^{p}$, and $b(s)>b^{p}$ for every $s>s^{p}$.

(ii) The bidding function $b(s)$ is strictly increasing in the range $\left(s^{p}, 1\right]$.

(iii) If $s^{p} \in(0,1)$, then the equilibrium action is $a\left(s, b(s), b^{p}\right)=r$ for $s>s^{p}$; and it is $a(s, b(s), p)=r$ for every $p \in\left(b^{p}, b(s)\right]$ for which there exists an $s^{\prime} \in S$ such that $b\left(s^{\prime}\right)=p$.

(iv) For $s \in\left[0, s^{p}\right), a\left(s, b(s), b^{p}\right)=l$.

Proof: see Appendix.

The characterization lemma essentially states that any nondecreasing equilibrium resembles the equilibrium that we constructed in the previous section for Theorem 1. More specifically, Lemma 1 shows that in any nondecreasing equilibrium, there is at most one interval, which includes zero, over which the bidding function is constant and equal to the pooling bid; outside of this interval, the bidding function is strictly increasing. Moreover, on the

\footnotetext{
${ }^{15} \mathrm{~A}$ straightforward modification of the lemma delivers a characterization of all nonincreasing equilibrium bidding functions as well.
} 
equilibrium path, the bidders who win an object choose $l$ if they have submitted the pooling bid, and choose $r$ if they have submitted a bid above the pooling bid. Below we provide an intuitive sketch of the argument for the characterization of nondecreasing equilibria when the auction is large (although Lemma 1 is stated for every market size).

- There must be pooling in any monotone equilibrium. Assume not, i.e., assume that the bidding function is strictly increasing. Consider a bidder who receives the signal zero. If this bidder's bid is equal to the auction price, then it must be the case that this bidder is almost certain that the state is $L$ if the auction is sufficiently large. By a continuity argument, this is also true for a bidder who receives signal $\epsilon>0$ for $\epsilon$ sufficiently close to zero. But then a bidder who receives signal zero must be willing to submit a bid that is greater than a bidder who receives signal $\epsilon$, because the bidder with signal zero is more convinced that the state is $L$ than the bidder with signal $\epsilon$. This, however, contradicts the assumption that the bidding function is strictly increasing.

- Bidders who bid a pooling bid choose action $l$ if they win an object. On the way to a contradiction, suppose that there is a bidder who bids the pooling bid and chooses action $r$ if he wins an object. Notice that he wins an object only when the auction price is not more than the pooling bid. Moreover, when the price is equal to the pooling bid, there is rationing with strictly positive probability. When the price is equal to the pooling bid, losing a unit is a signal more favorable to state $R$ because of the loser's curse. However, if this bidder deviates from such a strategy by increasings his bid slightly, he ensures that he wins an object whenever the auction price is equal to the pooling price. Such a deviation is profitable, because such a bidder chose action $r$ when he won an object before the deviation (by the hypothesis), and after the deviation, he wins an object in those instances when he had been losing by bidding the pooling bid.

- There is only one pooling bid. If the bidders who are bidding a pooling bid choose action $l$ when they win an object at the price equal to the pooling bid, then they also choose action $l$ if the price is lower than the pooling bid. Therefore, all bidders who are bidding below the pooling bid also choose action $l$. This is because such bidders must have received lower signals since we assume that the bidders use a nondecreasing bidding function. However, the bidder with signal zero then has the highest valuation for the object among all bidders whose bids are less than the highest pooling bid. This implies that the pooling bid chosen by a bidder who receives signal zero must be at least as large as all the other pooling bids. Therefore, our assumption that the bidders use a nondecreasing bidding function implies that there is at most one pooling bid.

- Bidders who submit bids above the pooling bid choose action $r$ if they win an object. Recall that $s^{p}$ is the highest signal for which $b\left(s^{p}\right)$ equals the pooling 
bid. Pick a signal $s^{\prime}$ greater than but arbitrarily close to $s^{p}$. Assume that a bidder who receives signal $s^{\prime}$ plays $l$ if he wins an object and the auction price is equal to the pooling bid. We now argue that this assumption leads to a contradiction. A bidder who receives signal $s^{\prime}$ prefers submitting a bid that exceeds the pooling bid to submitting the pooling bid because $s^{\prime}>s^{p}$. Now consider the bidder who receives signal zero. Suppose that this bidder deviates and submits a bid that exceeds the pooling bid by an arbitrarily small amount and wins an object at the pooling price. In this event, the posterior of a bidder with signal zero puts more weight on state $l$ than the posterior of a bidder with signal $s^{\prime}$. Therefore, if the bidder who receives signal $s^{\prime}$ prefers to submit a bid that exceeds the pooling bid, then so does a bidder who receives signal zero. However, this contradicts the fact that type zero submits the pooling bid.

In the theorem below, we use the characterization given by Lemma 1 to show that monotone equilibria cannot fully aggregate information. Moreover, we establish that a monotone equilibrium sequence exists if Condition 1 holds, i.e., if $\rho(0) \geq \rho^{*}$.

TheOREm 2 Suppose that Assumptions 1-4 hold. If $\boldsymbol{b}$ is nondecreasing, then $\boldsymbol{b}$ does not fully aggregate information. Moreover, if Condition 1 is satisfied, then a nondecreasing equilibrium sequence $\mathbf{b}$ exists.

Proof: We will prove the first part of the theorem here. The proof of the second part is in the Appendix.

Note that when $z$ is sufficiently large, a monotonic equilibrium bid function cannot be strictly increasing, because otherwise types that are arbitrarily close to zero would have a profitable deviation to submit bids of higher types. Therefore, such bid functions should have exactly one pooling bid, due to Lemma 1 . Let $b_{z}^{p}$ be the pooling bid, and let $s_{z}^{p}$ be the highest bidder type that bids the pooling bid in the monotonic bidding function $b_{z}$. Based on Lemma 1, our first observation is that:

$$
\rho\left(Y_{z-1}^{z \kappa} \leq s_{z}^{p}, s_{1}>s_{z}^{p}\right) \geq \rho^{*} .
$$

Let $s^{c} \in(0,1)$ be the unique signal such that

$$
F\left(s^{c} \mid L\right)^{1-\kappa}\left(1-F\left(s^{c} \mid L\right)\right)^{\kappa}=F\left(s^{c} \mid R\right)^{1-\kappa}\left(1-F\left(s^{c} \mid R\right)\right)^{\kappa} .
$$

We will now argue that for $\forall \varepsilon>0$, there exists a $Z_{\varepsilon}$ such that $s_{z}^{p}>s^{c}-\varepsilon$ for every $z>Z_{\varepsilon}$. The reason for the claim is the following: Suppose, on the contrary, that $s_{z}^{p}<s^{c}-\varepsilon$ for some $z>Z$ for every $Z$. Pick a convergent subsequence of such cutoffs, $s_{z}^{p}$, and renumerate 
its elements to cover the natural numbers. Then $\lim _{z \rightarrow \infty} \operatorname{Pr}\left(\omega=R \mid Y_{z-1}^{z \kappa} \leq s_{z}^{p}, s_{1}>s_{z}^{p}\right)<$ $\lim _{z \rightarrow \infty} \operatorname{Pr}\left(\omega=R \mid Y_{z-1}^{z \kappa}=s_{z}^{p}, s_{1}>s_{z}^{p}\right)=0$, which contradicts our initial observation that $\rho\left(Y_{z-1}^{z \kappa} \leq s_{z}^{p}, s_{1}>s_{z}^{p}\right) \geq \rho^{*}$.

Since $F\left(s^{c} \mid L\right)>1-\kappa$, by the Weak LLN it follows that $\lim _{z \rightarrow \infty} \operatorname{Pr}\left(p_{z}=b_{z}^{p} \mid L\right)=1$. Combining this with our initial observation that $\rho\left(Y_{z-1}^{z \kappa} \leq s_{z}^{p}, s_{1}>s_{z}^{p}\right) \geq \rho^{*}$, we argue that $\liminf _{z \rightarrow \infty} \operatorname{Pr}\left(p_{z}=b_{z}^{p} \mid R\right)>0$. Hence, the pooling price becomes the price with probability one conditional on the state of the world $L$, and with a nonnegligible probability conditional on the state of the world $R$. Therefore, the pooling price doesn't reveal the state of the world with a strictly positive probability in the limit as $z \rightarrow \infty$.

We now use the characterization in Lemma 1 to provide the intuition for why information is not fully aggregated in nondecreasing equilibria. The argument essentially shows that, in an arbitrarily large market, the cutoff bid $s^{p}$ is sufficiently large and therefore the price is equal to the pooling bid with probability one in state $L$ and with strictly positive probability in state $R$. We begin by supposing that in an auction with $n$ bidders and $n \kappa$ objects, the equilibrium bidding function is nondecreasing. Below we define the posterior likelihood ratio, for a bidder who receives signal $s$, if she wins an object and the auction price is equal to $b\left(s^{\prime}\right)$ where $b\left(s^{\prime}\right)>b^{p}$ :

$$
\begin{aligned}
\rho_{n}\left(p=b\left(s^{\prime}\right), s\right) & =\rho_{0} \frac{F\left(s^{\prime} \mid R\right)^{n-n \kappa-1}\left(1-F\left(s^{\prime} \mid R\right)\right)^{n \kappa-1} f(s \mid R) f\left(s^{\prime} \mid R\right)}{F\left(s^{\prime} \mid L\right)^{n-n \kappa-1}\left(1-F\left(s^{\prime} \mid L\right)\right)^{n \kappa-1} f(s \mid L) f\left(s^{\prime} \mid L\right)} \\
& =\rho_{0}\left(\frac{F\left(s^{\prime} \mid R\right)^{1-\kappa}\left(1-F\left(s^{\prime} \mid R\right)\right)^{\kappa}}{F\left(s^{\prime} \mid L\right)^{1-\kappa}\left(1-F\left(s^{\prime} \mid L\right)\right)^{\kappa}}\right)^{n} \frac{f(s \mid R) f\left(s^{\prime} \mid R\right)}{f(s \mid L) f\left(s^{\prime} \mid L\right)}\left(\frac{F\left(s^{\prime} \mid L\right)\left(1-F\left(s^{\prime} \mid L\right)\right)}{F\left(s^{\prime} \mid R\right)\left(1-F\left(s^{\prime} \mid R\right)\right)}\right) \\
& =\rho_{0}\left(\frac{g\left(F\left(s^{\prime} \mid R\right)\right)}{g\left(F\left(s^{\prime} \mid L\right)\right)}\right)^{n} \frac{f(s \mid R) f\left(s^{\prime} \mid R\right)}{f(s \mid L) f\left(s^{\prime} \mid L\right)}\left(\frac{F\left(s^{\prime} \mid L\right)\left(1-F\left(s^{\prime} \mid L\right)\right)}{F\left(s^{\prime} \mid R\right)\left(1-F\left(s^{\prime} \mid R\right)\right)}\right),
\end{aligned}
$$

where the function $g(t):=t^{(1-\kappa)}(1-t)^{\kappa}$. In words, the bidder's posterior likelihood is calculated in the event that she receives signal $s$, another bidder receives a signal $s^{\prime}$, there are $n \kappa-1$ other bidders who receive signals that exceed $s^{\prime}$, and there are $n-n \kappa-1$ other bidders who receive signals less than $s^{\prime}$. Let $s^{c}$ denote the unique signal $s^{\prime}$ such that $g\left(F\left(s^{\prime} \mid R\right)\right)=g\left(F\left(s^{\prime} \mid L\right)\right)$. See Figure 9 for a depiction of $s^{c}$. Note that if $s^{\prime}<s^{c}$, then $g\left(F\left(s^{\prime} \mid R\right)\right)<g\left(F\left(s^{\prime} \mid L\right)\right)$ (as depicted in Figure 9), and consequently, $\rho_{n}\left(p=b\left(s^{\prime}\right), s\right)$ converges to zero as $n$ grows large. ${ }^{16}$ We now provide the basic intuition behind the argument that establishes that information cannot be fully aggregated in a large auction:

- In state $L$, the probability that the auction price is equal to the pooling bid approaches one as the auction grows arbitrarily large. Note that, because

\footnotetext{
${ }^{16}$ Similarly, if $s^{\prime}>s^{c}$, then $g\left(F\left(s^{\prime} \mid R\right)\right)>g\left(F\left(s^{\prime} \mid L\right)\right)$ and consequently $\rho\left(p=b\left(s^{\prime}\right), s, n\right)$ converges to infinity as $n$ grows large.
} 


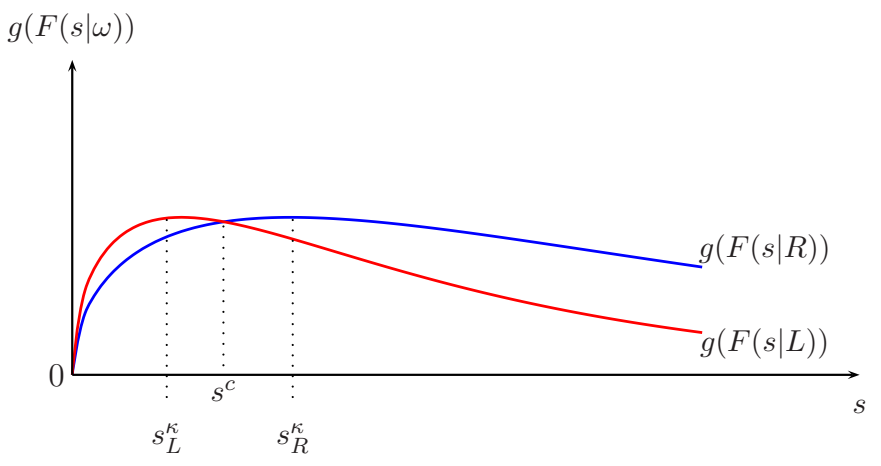

Figure 9: In this figure, $g(t):=t^{(1-\kappa)}(1-t)^{\kappa}$ and $s^{c}$ is the unique signal $s^{\prime}$ such that $g\left(F\left(s^{\prime} \mid R\right)\right)=$ $g\left(F\left(s^{\prime} \mid L\right)\right)$. The function $g(t)$ is concave and is maximized at $t^{*}=1-\kappa$. Recall that $s_{L}^{\kappa}$ and $s_{R}^{\kappa}$ are the unique signals such that $F\left(s_{L}^{\kappa} \mid L\right)=1-\kappa$ and $F\left(s_{R}^{\kappa} \mid R\right)=1-\kappa$, respectively. Note that weak MLRP and informativeness of signals together imply that $s_{L}^{\kappa}<s_{R}^{\kappa}$. Also, concavity of the function $g$ implies that $F\left(s^{c} \mid L\right)>s_{L}^{\kappa}$ and $F\left(s^{c} \mid R\right)<s_{R}^{\kappa}$.

of the law of large numbers, if $F\left(s^{p} \mid L\right)>1-\kappa$ (if the expected proportion of bidders who choose the pooling bid in state $L$ exceeds $1-\kappa)$, then the price is equal to the pooling bid with probability one. Assume that $F\left(s^{p} \mid L\right) \leq 1-\kappa$. This implies that $s^{p}<s^{c}$. However, this implication contradicts the findings of Lemma 1 that a bidder who submits a bid that exceeds the pooling bid plays $r$ when he wins an object at the price of the pooling bid. This is because $s^{p}<s^{c}$ implies that $\rho_{n}\left(p=b\left(s^{p}\right), s\right)$ converges to zero as $n$ grows large, i.e., his posterior probability that the state is $R$ converges to zero as the market grows large.

- The auction price is equal to the pooling bid with strictly positive probability when the state is $R$. Moreover, this probability is bounded strictly away from zero even as the number of bidders grows arbitrarily large. If this were not the case, then nobody would be willing to choose action $r$ when the price is equal to the pooling bid. However, we know from Lemma 1 that all the bidders who submit a bid above the pooling bid play $r$ when they win an object at the price of the pooling bid.

- Therefore, an outside observer is uncertain about the state when she observes that the auction price is equal to the pooling bid. Moreover, this event occurs with strictly positive probability.

4.1. Indeterminate and suppressed prices. In a monotone equilibrium, the price is equal to the pooling bid with probability arbitrarily close to one in state $L$. If the probability that the price is equal to the pooling bid approaches one also in state $R$, then the price reveals no information about the state of the world. Otherwise, the price is equal to the pooling bid in state $R$ with strictly positive probability. In this case, the lowest bid that exceeds the 
pooling bid is arbitrarily close to $v(r, R)$ in a large market because $s^{p}>s^{c}$. Therefore, if the price reveals some information, then it is either equal to the pooling bid or equal to $v(r, R)$ in state $\mathrm{R}$. Therefore, prices are not deterministic functions of the state of the world. Moreover, if $v(l, L)<v(r, R)$, then the price of the object is strictly less than its efficient use value with a strictly positive probability in state $R$. This is because the pooling bid cannot exceed $v(l, L)$ and the pooling bid is the auction price with strictly positive probability in state $R$.

\section{Discussion}

\subsection{Auctions with no Ex-Post Actions, Auctions with Actions, and Assumption}

1. In this subsection, we sketch how information is aggregated in an auction with actions where the valuation function does not satisfy Assumption 1 using ?'s methodology. We then contrast this sketch with the case where the valuation function satisfies Assumption 1.

For simplicity, suppose that $v(r, R)>v(l, L)=v(r, L)=v(l, R)=0$, that is, action $l$ is weakly dominated by action $r$. In this case, the valuation function $v$ satisfies neither inequality (1) nor (2) and our model coincides with ?'s model with two states of the world $\Omega=\{L, R\}$ where the value of the object is equal to zero in state $L$ and equal to $v(r, R)$ in state $R .^{17}$ In the unique symmetric equilibrium of the auction with $n$ bidders and $k$ objects, the bidding function is then as follows:

$$
b(s)=v(r, R) \operatorname{Pr}\left(\omega=R \mid s_{1}=s, Y_{n-1}^{k}=s\right)
$$

for every $s \in(0,1)$. This function is strictly increasing in $s$ because the signal distribution satisfies MLRP (see also Figure 10). ${ }^{18}$ Now consider the cutoff signal $s^{c} \in(0,1)$, which is the unique interior solution to the following equation: ${ }^{19}$

$$
F\left(s^{c} \mid L\right)^{1-\kappa}\left(1-F\left(s^{c} \mid L\right)\right)^{\kappa}=F\left(s^{c} \mid R\right)^{1-\kappa}\left(1-F\left(s^{c} \mid R\right)\right)^{\kappa} .
$$

If we keep $\kappa=\frac{k}{n}$ constant and take $n$ to infinity, then $\operatorname{Pr}\left(\omega=R \mid s_{1}=s, Y_{n-1}^{k}=s\right)$ approaches zero for any $s<s^{c}$. Moreover, $\operatorname{Pr}\left(\omega=R \mid s_{1}=s, Y_{n-1}^{k}=s\right)$ approaches one for any $s>s^{c}$. Therefore, the bid of any bidder who receives a signal smaller than $s^{c}$ approaches zero, and the bid of any bidder who receives a signal that exceeds $s^{c}$ approaches $v(r, R)$. A

\footnotetext{
${ }^{17}$ More generally, if the valuation function does not satisfy inequality (1), or (2), then the model in this paper coincides with ?'s model with two states of the world $\Omega=\{L, R\}$ and the arguments provided here and in ? can be used to show that information is aggregated in any symmetric equilibrium of the auction as the market grows arbitrarily large.

${ }^{18}$ This function is strictly increasing in $s$ even if the signal distribution satisfies only weak MLRP.

19 The uniqueness follows from the following: weak MLRP taken together with the assumption that signals are not completely uninformative implies that for any $s \in(0,1), F\left(s^{c} \mid L\right)>F\left(s^{c} \mid R\right)$. The function $t(q)=q^{1-\kappa}(1-q)^{\kappa}$ is single-peaked and has a maximum at $q=1-\kappa$. Therefore, the solution is unique.
} 


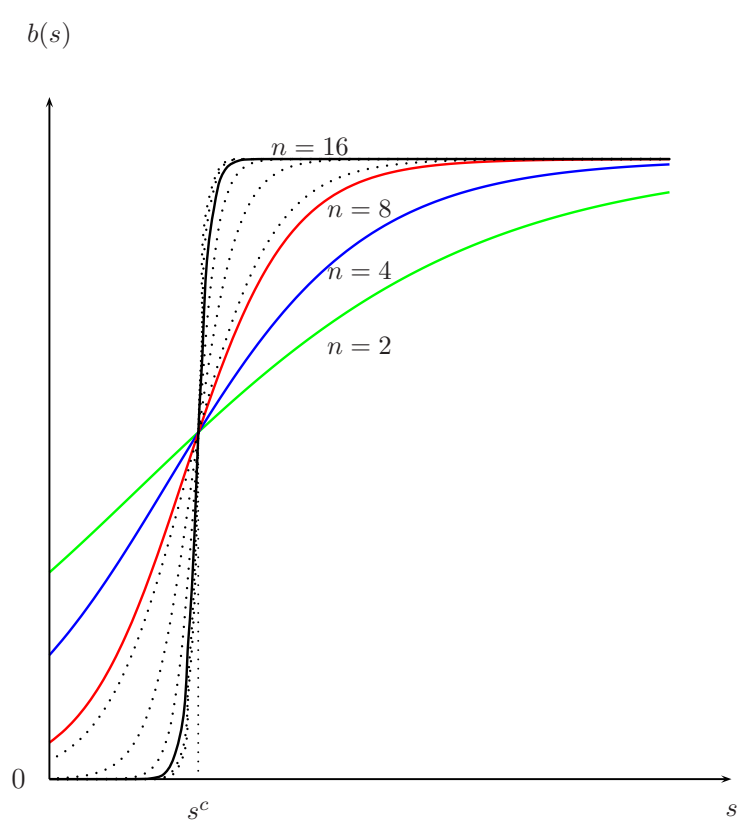

Figure 10: This figure depicts the equilibrium bidding function in ? for $\kappa=1 / 2$ for different market sizes. For every $\epsilon>0$, the range of signals $\left[\underline{s}_{n}, \bar{s}_{n}\right]$ whose bids are in the interval $[\epsilon, v(r, R)-\epsilon]$ shrinks to the point $s^{c}$.

bidder with signal $s^{c}$ always wins an object in state $L$ and never wins an object in state $R$. Therefore, the auction price approaches zero in state $L$ and $v(r, R)$ in state $R$ as $n$ grows large. Since prices are different in each of the two states of the world, an outside observer can learn the state simply by observing the price.

Alternatively, suppose that the valuation function satisfies Assumption 1, i.e, that $v(l, L)>$ 0. In this case, Lemma 1 implies that all types who bid strictly above the pooling bid take action $r$ at any price. We first look at types who are arbitrarily close to the cutoff signal $s^{p}$ and are bidding above the pooling bid $b^{p}$. Since they are taking action $r$ at prices close to their bids, the following holds:

$$
\rho\left(\omega=R \mid s_{1}>s^{p}, Y_{n-1}^{k} \leq s^{p}\right)>\rho^{*} .
$$

Therefore, for every $\epsilon>0$, if $n$ is sufficiently large, it has to be the case that $s^{p}$ is at least $s^{c}-\epsilon$. Therefore, the price becomes the pooling bid in state $L$ with probability close to one when $n$ is sufficiently large. On the other hand, bidders with signals close to but above $s^{p}$ take action $r$ when the price is $b^{p}$. Therefore, as $n$ gets larger, it must be the case that the price is the pooling bid with a non vanishing probability conditional on state $R$. And hence, the pooling bid does not reveal the state of the world even when the market is very large. The equilibrium price distribution becomes concentrated on two points: one point at the 
pooling bid and another at $v(r, R)$.

5.2. Sketch of the Construction with Finite Markets. In this subsection, we also assume that $f(. \mid R)$ and $f(. \mid L)$ are continuous functions. For every market size $z$, we first find a cutoff signal $s_{z}^{p} \in(0,1)$ such that the following holds:

$v(r, R) \operatorname{Pr}\left(\omega=R \mid s_{1}=s_{z}^{p}, Y_{z-1}^{\kappa z} \in\left[0, s_{z}^{p}\right)\right)=v(l, L) \operatorname{Pr}\left(\omega=L \mid s_{1}=s_{z}^{p}, Y_{z-1}^{\kappa z} \in\left[0, s_{z}^{p}\right), 1\right.$ wins by $\left.b_{z}^{p}\right)$.

If the bidding function satisfies the following: $i$ ) it is constant and is equal to some arbitrary bid $b_{z}^{p}$ over the interval $\left.\left[0, s_{z}^{p}\right) ; i i\right)$ it is strictly increasing in the interval $\left(s_{z}^{p}, 1\right]$; and $\left.i i i\right)$ it is above $b_{z}^{p}$ in the interval $\left(s_{z}^{p}, 1\right]$, then the valuation for the object of a bidder with signal $s_{z}^{p}$ is identical under the following two cases: $i$ ) if he bids $b_{z}^{p}$ and wins an object $i i$ ) if he submits a bid above $b_{z}^{p}$, wins an object, and the auction price is equal to $b_{z}^{p}$.

REMARK 6 Note that from the loser's curse, it follows that:

$$
\operatorname{Pr}\left(\omega=R \mid s_{1}=s_{z}^{p}, Y_{z-1}^{\kappa z} \in\left[0, s_{z}^{p}\right)\right)>\operatorname{Pr}\left(\omega=R \mid s_{1}=s_{z}^{p}, Y_{z-1}^{\kappa z} \in\left[0, s_{z}^{p}\right), 1 \text { wins by } b_{z}^{p}\right) .
$$

Therefore, for the valuation for the object of a bidder who receives signal $s_{z}^{p}$ to be identical in both instances, the following two inequalities must hold:

$$
\rho\left(\omega=R \mid s_{1}=s_{z}^{p}, Y_{z-1}^{\kappa z} \in\left[0, s_{z}^{p}\right)\right)>\rho^{*}
$$

and

$$
\rho\left(\omega=R \mid s_{1}=s_{z}^{p}, Y_{z-1}^{\kappa z} \in\left[0, s_{z}^{p}\right), 1 \text { wins by } b_{z}^{p}\right)<\rho^{*} .
$$

In other words, the bidder who receives signal $s_{z}^{p}$ should be taking action $l$ when he wins by bidding the pooling bid, and action $r$ when he wins with a higher bid.

The existence of such a cutoff is guaranteed when $z$ is sufficiently large because of the intermediate value theorem. Note that the term given just below is strictly negative if $s$ is close to zero and if $z$ is larger than a certain market size $Z_{1}$; and it is strictly positive if $s$ is close to one and $z$ is larger than a certain market size $Z_{2}$.

$h_{z}(s):=v(r, R) \operatorname{Pr}\left(\omega=R \mid s_{1}=s, Y_{z-1}^{\kappa z} \in[0, s)\right)-v(l, L) \operatorname{Pr}\left(\omega=L \mid s_{1}=s, Y_{z-1}^{\kappa z} \in[0, s), 1\right.$ wins by $\left.b_{z}^{p}\right)$.

Moreover, $h_{z}(s)$ is strictly increasing in $s$ and is continuous. Hence, when the market size $z$ is larger than $\max \left\{Z_{1}, Z_{2}\right\}$, there is a unique $s_{z}^{p}$ that satisfies the equality $h_{z}\left(s_{z}^{p}\right)=0$. 
We set the pooling bid $b_{z}^{p}$ equal to the valuation of a bidder who receives signal $s_{z}^{p}$ conditional on winning the object at a price equal to the pooling price, i.e.,

$$
b_{z}^{p}=v(r, R) \operatorname{Pr}\left(\omega=R \mid s_{1}=s_{z}^{p}, Y_{z-1}^{\kappa z} \in\left[0, s_{z}^{p}\right)\right),
$$

and we complete the bidding function a-la ? for signals higher than $s_{z}^{p}$. That is, for $s>s_{z}^{p}$ the bidding function is defined as follows:

$$
\left.b_{z}(s)=v(r, R) \operatorname{Pr}\left(\omega=R \mid s_{1}=s, Y_{z-1}^{\kappa z}=s\right)\right) .
$$

Note that for $s>s_{z}^{p}$, the following equality holds:

$$
\left.b_{z}(s)=v(r, R) \operatorname{Pr}\left(\omega=R \mid s_{1}=s, Y_{z-1}^{\kappa z}=s\right)\right)>v(r, R) \operatorname{Pr}\left(\omega=R \mid s_{1}=s_{z}^{p}, Y_{z-1}^{\kappa z} \in\left[0, s_{z}^{p}\right)\right)=b_{z}^{p} .
$$

Bidders with signals higher than $s_{z}^{p}$ can not profitably deviate because they weakly prefer to bid above $b_{z}^{p}$. Agents with signals lower than $s_{s}^{p}$ would clearly not want to submit a bid that exceeds $b_{z}^{p}$ and to take action $r$ upon winning the object at some price. Therefore, the only deviation left to check is the deviation where a bidder with a signal lower than $s_{s}^{p}$ bids above $b_{z}^{p}$ and takes action $l$ upon winning the object at a price equal to $b_{z}^{p}$. The bidder who would have the most to gain from such a deviation is a bidder who receives signal zero. As we will explain below, our two restrictions on the priors and values ensure that such a deviation is not profitable and that the cutoff types converge to a signal above $s_{R}^{\kappa}$.

To see that a bidder with signal zero does not want to deviate, we look at the three possible limit points of $s_{z}^{p}$ and argue that the bidder does not want to bid above $b_{z}^{p}$ and take action $l$ upon winning the object in any of the three cases. Moreover, we will argue that any such limit point has to be at least $s_{R}^{\kappa}$.

First, suppose that a subsequence of the cutoffs converges to a signal strictly higher than $s_{R}^{\kappa}$. If this is the case, then the pooling bid reveals no information when the market is sufficiently large. Therefore, a bidder with signal zero who deviates and submits a bid above the pooling bid and wins an object at a price equal to the pooling bid would prefer to take action $r$ because $\rho(0)>\rho^{*}$ and because the pooling bid reveals no information. Since the bid $b_{z}^{p}$ is equal to the valuation of the bidder with signal $s_{z}^{p}$ when the price is equal $b_{z}^{p}$, the bidder with signal zero would have a negative payoff under such a deviation.

Second, suppose that a subsequence of the cutoffs converges to $s_{R}^{\kappa}$. We will now argue that this cannot be the case. If this were the case, then the critical observation is that, in state $R$, the fraction of objects remaining to be allocated to those bidders bidding the pooling bid approaches zero. Moreover, the fraction of objects to be shared among those bidders in state 
$L$ approaches a strictly positive number. Therefore, for a bidder who bids the pooling bid, winning an object is very strong evidence for the state $L$. The pooling bid thus approaches $v(l, L)$ as the market gets large. But this contradicts the property satisfied in the choice of the cutoff signal that, the bidder with the cutoff signal is indifferent between bidding the pooling bid and bidding above the pooling bid, because we assumed that $u\left(\rho\left(s_{R}^{\kappa}\right)\right)<v(l, L)$.

Third, suppose that a subsequence of the cutoffs converges to a signal strictly less than $s_{R}^{\kappa}$. In this case, as the market gets large, the pooling price is increasingly likely to reveal that the state is $L$. However, it cannot then be the case that the posterior of the bidder with signal $s_{z}^{p}$ puts a sufficiently high probability on state $R$ when the bidder bids above $b_{z}^{p}$ and the price is the pooling bid. ${ }^{20}$ Therefore, a subsequence of the cutoffs cannot converge to a signal which is strictly less than $s_{R}^{\kappa}$.

\section{Conclusion}

In this paper, we have explored the role of market prices in aggregating information about the correct use of objects. In our set-up, multiple homogeneous goods are allocated among multiple bidders via a Vickrey-type auction. Our main finding is that, when prices contain information about the ex-post actions that the owners of the object will take, then prices may not reveal all the information available in the market. In the extreme case, prices reveal no information about the state of the world, and a nonnegligible fraction of the objects are thus used incorrectly.

There are trivial mechanisms that could aggregate information. In our model, there is no room for allocative inefficiency. Therefore, a direct mechanism that elicits the signals of bidders, allocates the objects randomly, discloses the signal profile would achieve full efficiency. However, we study Vickrey auctions for four main reasons. First, such auctions are frequently used in practice. Treasury bill auctions are prominent examples. Second, such auctions resemble competitive markets in which agents are price takers, since in a Vickrey auction, a bidder cannot change the price he pays for the object by altering his bid. Third, just as in competitive markets, there is a uniform price. Fourth, there is a large body of academic work that studies Vickrey auctions. Therefore, knowing how such auctions perform when the objects have a use value which depends on ex-post actions is in and of itself.

We interpret our results as suggesting that it is too much to expect prices alone to reveal the state of the world perfectly. Also, our results highlight that markets have several statistics other than price, such as the amount of rationing, volume of trade, and bid distributions,

20 More precisely, this contradicts the fact that

$$
\rho\left(\omega=R \mid s_{1}=s_{z}^{p}, Y_{z-1}^{\kappa z} \in\left[0, s_{z}^{p}\right)\right)>\rho^{*} .
$$


that are relevant for aggregating information.

\section{A. Organization of the Appendix}

We start by proving Theorem 2 instead of Theorem 1, because the construction we use for the former is used for the latter theorem. Later we prove Lemma 1, and then we present the proofs of some lemmata that we use in the proofs of our theorems and lemma 1.

\section{B. Proof of Theorem 2}

Theorem 2. Suppose that Assumptions 1-4 hold. If $\boldsymbol{b}$ is nondecreasing, then $\boldsymbol{b}$ does not fully aggregate information. Moreover, if Condition 1 is satisfied, then a nondecreasing equilibrium sequence $\mathbf{b}$ exists.

The first part of the theorem is proven in the main text. Here we prove the second part. We remind that, the informative signal assumption is assumed to hold, and hence $f(0 \mid L) \neq$ $f(0 \mid R)$.

B.1. Method used for the construction The construction has two general steps. In the first step, we show that in a large market with size $z$, there exists a cutoff signal, $s_{z}^{p}$ such that in a monotonic bidding profile $b_{z}$ where all types below $s_{z}^{p}$ bid a pooling bid, the following two properties are satisfied. i) the value of the object to bidders with signals $s<s_{z}^{p}$, who win a unit by bidding the pooling bid, is not less than the value of the object to such bidders if they win a unit by bidding above the pooling bid, and when the price is the pooling bid. ii) The value of the object to bidders with signals $s>s_{z}^{p}$ when they bid above the pooling bid and the price is the pooling bid is not less than if such bidders bid the pooling bid and win a unit. In this step, we also determine the value of the pooling bid.

The second step of the construction shows that under condition 1, the bidding profiles constructed in step 1 constitute an equilibrium of the auction game when $z$ is sufficiently large. We do this by showing that no type has a profitable deviation from the bidding profile constructed in step 1.

B.2. Step 1: Cutoff type For any $s \in(0,1), s^{\prime} \in S$ and $z \in \mathbb{Z}$, let $\rho_{z}^{-}\left(s^{\prime}, s\right)$ and $\rho_{z}^{+}\left(s^{\prime}, s\right)$ be

$$
\begin{aligned}
& \rho_{z}^{-}\left(s^{\prime}, s\right):= \frac{P\left(Y_{z-1}^{\kappa z} \leq s, s_{1}=s^{\prime}, 1 \text { wins the lottery } \mid R\right)}{P\left(Y_{z-1}^{\kappa z} \leq s, s_{1}=s^{\prime}, 1 \text { wins the lottery } \mid L\right)}, \\
& \rho_{z}^{+}\left(s^{\prime}, s\right):=\frac{P\left(Y_{z-1}^{\kappa z} \leq s, s_{1}=s^{\prime} \mid R\right)}{P\left(Y_{z-1}^{\kappa z} \leq s, s_{1}=s^{\prime} \mid L\right)},
\end{aligned}
$$

The event that " 1 wins the lottery" corresponds to the event that 1 wins a prize (or equivalently one unit of the object) in the following auxiliary lottery whose odds depend on the signal distribution across the bidders. The lottery has $q$ prizes allocated equally likely to $O$ 
people, where the number of prizes $q=\max \left\{0, \kappa z-\left|j \in\{2, \ldots, z\}: s_{j}>s\right|\right\}$ and the number of people is $o=1+\left|j \in\{2, \ldots, z\}: s_{j} \leq s\right|{ }^{21}$ Intuitively, $\rho_{z}^{-}\left(s^{\prime}, s\right)$ is the posterior likelihood ratio of state $R$ and $L$ for type $s^{\prime}$, when he bids the pooling bid and wins a unit, where the bidders who bid the pooling bid are those with signals less than $s$. The second function, $\rho_{z}^{+}\left(s^{\prime}, s\right)$ is the posterior likelihood ratio of states $R$ and $L$ when a bidder with a signal $s^{\prime}$ wins a unit by bidding above the pooling bid, at a price equal to the pooling bid.

REMARK 7 Observe that, these functions are continuous in their second argument. This is because the cdf $F(s \mid \omega)$ admits a positive density function $f$ and is thus a continuous function of $s$. On the other side, if $f$ is a continuous function, then these functions are also continuous in their first argument. However, we have not made such an assumption, hence these functions may have jumps as we vary the first argument.

We also make the following definitions:

$$
\begin{aligned}
& \rho_{z}^{-}(s):=\frac{P\left(Y_{z-1}^{\kappa z} \leq s, s_{1}=s, 1 \text { wins the lottery } \mid R\right)}{P\left(Y_{z-1}^{\kappa z} \leq s, s_{1}=s, 1 \text { wins the lottery } \mid L\right)}, \\
& \rho_{z}^{+}(s):=\frac{P\left(Y_{z-1}^{\kappa z} \leq s, s_{1}=s \mid R\right)}{P\left(Y_{z-1}^{\kappa z} \leq s, s_{1}=s \mid L\right)}
\end{aligned}
$$

Intuitively, these functions are the posterior likelihoods of the states for the cutoff types.

REMARK 8 From Pesendorfer and Swinkels (Lemma 7, page 1272) and from $f(0 \mid L) \neq$ $f(0 \mid R)$ we know that $i) \rho_{z}^{-}(s)<\rho_{z}^{+}(s)$ for any $s \in(0,1)$, and ii) $\rho_{z}^{-}\left(s^{\prime}, s\right)<\rho_{z}^{+}\left(s^{\prime}, s\right)$ for any $s, s^{\prime} \in(0,1)$. This is called the loser's curse. Moreover, both of these functions are strictly increasing in s and weakly increasing in s' since $f(0 \mid L) \neq f(0 \mid R)$, as we later show in Lemma 6.

Note that, because $\rho_{z}^{-}$and $\rho_{z}^{+}$are both increasing functions, $u\left(\rho_{z}^{-}\right)$and $u\left(\rho_{z}^{+}\right)$are both at most single-troughed functions. Now we make two observations about the endpoints of the functions $\rho_{z}^{-}$and $\rho_{z}^{+}$. Our third observation lets us draw the shapes of the functions $u\left(\rho^{-}\right)$ and $u\left(\rho^{+}\right)$.

\section{LEMMA 2}

1. $\exists \varepsilon>0$ and a $Z_{1}$ such that $\rho_{z}^{-}(s)<\rho^{*}$ and $\rho_{z}^{+}(s)<\rho^{*}$ for every $s \leq \varepsilon$ and every $z>Z_{1}$.

2. $\exists \varepsilon>0$ and a $Z_{2}$ such that $\rho_{z}^{-}(s)>\rho^{*}$ and $\rho_{z}^{+}(s)>\rho^{*}$ for every $s \geq 1-\varepsilon$ and every $z>Z_{2}$.

\footnotetext{
${ }^{21}$ The index sets exclude the number 1 since it is reserved for the bidder who is doing these calculations for her best response.
} 


\section{Proof:}

1. $\exists \varepsilon>0$ such that $\lim _{z \rightarrow \infty} \rho_{z}^{-}(\varepsilon)=\lim _{z \rightarrow \infty} \rho_{z}^{+}(\varepsilon)=0$. Since $\rho_{z}^{-}(s)<\rho_{z}^{+}(s)$ for $s \in(0,1)$, we have that $u\left(\rho_{z}^{-}(s)\right)>u\left(\rho_{z}^{+}(s)\right)$ for $s \leq \varepsilon$.

2. This is because, $\exists \varepsilon>0$ such that $\lim _{z \rightarrow \infty} \rho_{z}^{-}(1-\varepsilon)=\lim _{z \rightarrow \infty} \rho_{z}^{+}(1-\varepsilon)=\rho_{0}(1-\varepsilon)>\rho^{*}$, by condition 1 . Since $\rho_{z}^{-}$and $\rho_{z}^{+}$are strictly increasing, we have that $\rho_{z}^{-}(s)>\rho^{*}$ and $\rho_{z}^{+}(s)>\rho^{*}$ for every $s \geq 1-\varepsilon$ when $z$ is sufficiently large. Since $\rho_{z}^{-}(s)<\rho_{z}^{+}(s)$ for $s<1$, we have that $u\left(\rho_{z}^{-}(s)\right)<u\left(\rho_{z}^{+}(s)\right)$ for $s \geq 1-\varepsilon$.

\section{Lemma 3}

1. For every $z>\max \left\{Z_{1}, Z_{2}\right\}$, there is a unique $s_{z}^{p} \in(\varepsilon, 1-\varepsilon)$ that satisfies the following: $u\left(\rho_{z}^{-}(s)\right)-u\left(\rho_{z}^{+}(s)\right)<0$ for $s>s_{z}^{p}$ and $u\left(\rho_{z}^{-}(s)\right)-u\left(\rho_{z}^{+}(s)\right)>0$ for $s<s_{z}^{p}$.

2. When such an $s_{z}^{p}$ exists, $\rho_{z}^{+}(s)>\rho^{*}$ for $s>s_{z}^{p}$ and $\rho_{z}^{-}(s)<\rho^{*}$ for $s<s_{z}^{p}$.

PROOF: 1. Let $s_{z}^{1}:=\sup _{s}\left\{\rho_{z}^{-}(s) \leq \rho^{*}\right\}$ and $s_{z}^{2}:=\sup _{s}\left\{\rho_{z}^{+}(s) \leq \rho^{*}\right\}$. Note that $s_{z}^{1}, s_{z}^{2} \in$ $(0,1)$ when $z>\max \left\{Z_{1}, Z_{2}\right\}$ from lemma 2. Moreover, because $\rho_{z}^{-}(s)<\rho_{z}^{+}(s), z_{1} \geq z_{2}$. In the range $\left[0, z_{2}\right), u\left(\rho_{z}^{-}(s)\right)>u\left(\rho_{z}^{+}(s)\right)$, and in the range $\left(z_{1}, 1\right], u\left(\rho_{z}^{+}(s)\right)>u\left(\rho_{z}^{-}(s)\right)$.

If $z_{1}=z_{2}$, then $s_{z}^{p}=z_{1}=z_{2}$ satisfies the claim of the lemma. So now we assume that $z_{1}>z_{2}$.

In the range $\left(z_{2}, z_{1}\right), u\left(\rho_{z}^{-}(s)\right)$ is strictly decreasing and $u\left(\rho_{z}^{+}(s)\right)$ is strictly increasing. Therefore, $u\left(\rho_{z}^{-}(s)\right)-u\left(\rho_{z}^{+}(s)\right)$ is strictly negative in $\left[0, z_{2}\right)$, strictly increasing in $\left[z_{2}, z_{1}\right]$, and strictly positive in $\left(z_{1}, 1\right]$. Therefore, by the intermediate value theorem, there is a unique signal $s_{z}^{p}$, in the range $\left[z_{2}, z_{1}\right]$ that satisfies the property in the lemma.

2. As we have argued, such a signal is in the range $\left[z_{2}, z_{1}\right]$ and hence $\rho_{z}^{+}(s)>\rho^{*}$ for $s>s_{z}^{p}$ and $\rho_{z}^{-}(s)<\rho^{*}$ for $s<s_{z}^{p}$.

B.3. Setting the pooling bid and its properties We now determine the bidding function, $b_{z}$, when $z>\max \left\{Z_{1}, Z_{2}\right\}$. The bidding function is constant and equal to a number that is denoted by $b_{z}^{p}$ in the interval $\left[0, s_{z}^{p}\right)$ and is strictly increasing and equal to $u\left(\rho\left(s_{1}=s, Y_{z-1}^{\kappa z}=s\right)\right)$ in the region $\left(s_{z}^{p}, 1\right)$. Notice that, the increasing part of the bidding function coincides with Pesendorfer and Swinkel's equilibrium bidding function, in the case where bidders are taking action $r$.

Next, we determine the value of $b_{z}^{p}$.

$$
b_{z}^{p}=\min \left\{\inf _{s<s_{z}^{p}} u\left(\rho_{z}^{-}(s)\right), \inf _{s>s_{z}^{p}} u\left(\rho_{z}^{+}(s)\right)\right\}
$$

ReMARK 9 A few comments are now in order: 
1) Since $\rho_{z}^{-}(s)<\rho^{*}$ for $s<s_{z}^{p}$, as shown in lemma 3, and since $\rho_{z}^{-}$is an increasing function, $u\left(\rho_{z}^{-}(s)\right)$ is decreasing for $s<s_{z}^{p}$.

2) $\inf _{s<s_{z}^{p}} u\left(\rho_{z}^{-}(s)\right)=\lim _{s \nearrow s_{z}^{p}} u\left(\rho_{z}^{-}(s)\right)$, because $u\left(\rho_{z}^{-}(s)\right)$ is a decreasing function with a bounded range, hence its limit exists and is equal to the inf expression. Moreover, since $\rho_{z}^{-}\left(s^{\prime}, s\right)$ is continuous in its second argument, we have that $\inf _{s<s_{z}^{p}} u\left(\rho_{z}^{-}(s)\right)=\inf _{s<s_{z}^{p}} u\left(\rho_{z}^{-}\left(s, s_{z}^{p}\right)\right)$.

3) Similar to item 2) above, $\inf _{s>s_{z}^{p}} u\left(\rho_{z}^{+}(s)\right)=\inf _{s>s_{z}^{p}} u\left(\rho_{z}^{+}\left(s, s_{z}^{p}\right)\right)$.

4) If $f(s \mid \omega)$ is continous at $s_{z}^{p}$, then $b_{z}^{p}=u\left(\rho_{z}^{-}\left(s_{z}^{p}\right)\right)=u\left(\rho_{z}^{+}\left(s_{z}^{p}\right)\right)$.

In the next step we will show that there is a $Z_{3}$ such that for any $z>Z_{3}$ a bidding function $b_{z}$ that is characterized by the cutpoint $s_{z}^{p}$ and the pooling bid $b_{z}^{p}$ as defined above is an equilibrium.

We state the following remark that summarizes some of the findings up to now, before we proceed:

REMARK 10 The posterior likelihood ratio of types lower than $s_{z}^{p}$, conditional on winning at price $b_{z}^{p}$ is less than $\rho^{*}$, and types higher than $s_{z}^{p}$, conditional on the price being $b_{z}^{p}$ has a posterior likelihood ratio that is more than $\rho^{*}$. In particular,

$$
\begin{aligned}
& \rho\left(s_{1}=s, Y_{z-1}^{\kappa z} \leq s_{z}^{p}, 1 \text { wins with } b_{z}^{p}\right) \leq \rho^{*} \text { for } s<s_{z}^{p}, \text { and } \\
& \rho\left(s_{1}=s, Y_{z-1}^{\kappa z} \leq s_{z}^{p}\right) \geq \rho^{*} \text { for } s>s_{z}^{p} .
\end{aligned}
$$

The reason is that, as we have shown in lemma 3, $\rho_{z}^{-}(s)<\rho^{*}$ for $s<s_{z}^{p}$. Since $\rho_{z}^{-}\left(s, s_{z}^{p}\right)=$ $\lim _{s^{\prime} \uparrow s_{z}^{p}} \rho_{z}^{-}\left(s, s^{\prime}\right) \leq \sup _{s^{\prime} \uparrow s_{z}^{p}} \rho_{z}^{-}\left(s^{\prime}\right) \leq \rho^{*}$, we have established the first inequality. A similar reasoning yields the second inequality.

B.4. Step 2: Checking deviations In this step, we will show that the bid function we constructed in step 1 (i.e., $b_{z}$ ) is an equilibrium when $z$ is large (i.e., when $z>Z_{3}$ for some integer $Z_{3}$ ). In particular, we will show that no type has a profitable deviation. In the following we assume that $z$ is large enough that $s_{z}^{p}$ exists, i.e., $z>\max \left\{Z_{1}, Z_{2}\right\}$.

B.4.1. Bidders with signals above $s_{z}^{p}$ Pick a type $s>s_{z}^{p}$. We will now argue that, such a type $s$ wouldn't have a profitable deviation to any bid between $b_{z}^{p}$ and $b(s)$. Pick a type $s^{\prime}$ that satisfies $s_{z}^{p}<s^{\prime}<s$. Then, $u\left(\rho\left(s_{1}=s, Y_{z-1}^{\kappa z}=s^{\prime}\right)\right) \geq b_{z}\left(s^{\prime}\right)=u\left(\rho\left(s_{1}=s^{\prime}, Y_{z-1}^{\kappa z}=s^{\prime}\right)\right)$, because $\rho\left(s_{1}=s, Y_{z-1}^{\kappa z}=s^{\prime}\right) \geq \rho\left(s_{1}=s^{\prime}, Y_{z-1}^{\kappa z}=s^{\prime}\right) \geq \rho^{*}$. Similarly such types wouldn't have a positive payoff conditional on prices above their bid, hence they wouldn't have a profitable deviation to bid above $b(s)$. Finally, in the next paragraph, we will argue that such a type doesn't find it a profitable deviation to bid at $b_{z}^{p}$.

In order to prove that bidders with signals above $s_{z}^{p}$ don't have profitable deviations to 
bid $b_{z}^{p}$, we will show two inequalities for every $s>s_{z}^{p}$ :

$$
\begin{aligned}
& u\left(\rho_{z}^{+}\left(s, s_{z}^{p}\right)\right) \geq b_{z}^{p} \\
& u\left(\rho_{z}^{+}\left(s, s_{z}^{p}\right)\right) \geq u\left(\rho_{z}^{-}\left(s, s_{z}^{p}\right)\right)
\end{aligned}
$$

To see that these inequalities suffice to prove that bidding $b_{z}^{p}$ is not a profitable deviation, notice that the first inequality says that, bidding above $b_{z}^{p}$ gives a non-negative payoff at prices equal to $b_{z}^{p}$. The second inequality says that, the payoff to a bidder when he bids above $b_{z}^{p}$ and the price is $b_{z}^{p}$ is not less than when he bids $b_{z}^{p}$ and wins a unit. Moreover, the probability of winning a unit by bidding above $b_{z}^{p}$ is strictly larger than winning by bidding $b_{z}^{p}$. Hence, bidding $b_{z}^{p}$ is not a profitable deviation. Next, we will prove the two inequalities.

Before proving these two inequalities remember that a property of type $s_{z}^{p}$ is that $u\left(\rho_{z}^{-}(s)\right)<$ $u\left(\rho_{z}^{+}(s)\right)$ and $\rho_{z}^{+}(s)>\rho^{*}$ for every $s>s_{z}^{p}$. We will now start showing the first inequality. Since $\rho^{+}(s) \geq \rho^{*}$ for every $s>s_{z}^{p}$, and since $\rho^{+}(.,$.$) is continuous in its second argument, we$ have that:

$$
\inf _{s>s_{z}^{p}} \rho^{+}\left(s, s_{z}^{p}\right)=\inf _{s>s_{z}^{p}} \rho^{+}(s) \geq \rho^{*}
$$

Since $\rho^{+}(.,$.$) is increasing in its first argument, we have that, for every s>s_{z}^{p}$ :

$$
\rho^{+}\left(s, s_{z}^{p}\right) \geq \inf _{s>s_{z}^{p}} \rho^{+}\left(s, s_{z}^{p}\right) \geq \rho^{*}
$$

Since $b_{z}^{p} \leq \inf _{s>s_{z}^{p}} u\left(\rho^{+}\left(s, s_{z}^{p}\right)\right)$, and since $u(\rho)$ is increasing when $\rho \geq \rho^{*}$, we have that $u\left(\rho^{+}\left(s, s_{z}^{p}\right)\right) \geq b_{z}^{p}$, which establishes the first inequality.

Now we will show the second inequality. There are two cases to consider. Either $\rho^{-}\left(s, s_{z}^{p}\right) \geq$ $\rho^{*}$ or $\rho^{-}\left(s, s_{z}^{p}\right)<\rho^{*}$. In the former case, $\rho^{+}\left(s, s_{z}^{p}\right) \geq \rho^{-}\left(s, s_{z}^{p}\right)$ together with the facts that both are at least $\rho^{*}$ and $u$ is increasing when $\rho \geq \rho^{*}$ deliver the desired inequality. We will now show the validity of the inequality in the latter case.

On the way to a contradiction, suppose that $u\left(\rho^{+}\left(s, s_{z}^{p}\right)\right)<u\left(\rho^{-}\left(s, s_{z}^{p}\right)\right)$ and that $\rho^{-}\left(s, s_{z}^{p}\right)<$ $\rho^{*}$. Since $\rho^{-}(.,$.$) is continuous in its second argument, and since u(\rho)$ is decreasing when $\rho<\rho^{*}$ and $\rho^{-}$is increasing in both of its arguments, there exists a signal $s^{\prime}>s_{z}^{p}$ and an $\epsilon>0$ such that $u\left(\rho^{+}\left(s, s_{z}^{p}\right)\right)<u\left(\rho^{-}\left(s^{\prime \prime}\right)\right)-\epsilon$ for every $s^{\prime \prime} \in\left(s_{z}^{p}, s^{\prime}\right]$. Since $u\left(\rho^{-}(s)\right)<u\left(\rho^{+}(s)\right)$ for every $s>s_{z}^{p}, u\left(\rho^{+}\left(s, s_{z}^{p}\right)\right)<u\left(\rho^{+}\left(s^{\prime \prime}\right)\right)-\epsilon$ for every $s^{\prime \prime} \in\left(s_{z}^{p}, s^{\prime}\right]$. Therefore, $u\left(\rho^{+}\left(s, s_{z}^{p}\right)\right)<$ $\inf _{s^{\prime \prime \prime}>s_{z}^{p}} u\left(\rho^{+}\left(s^{\prime \prime \prime}\right)\right)-\epsilon=\inf _{s^{\prime \prime \prime}>s_{z}^{p}} u\left(\rho^{+}\left(s^{\prime \prime \prime}, s_{z}^{p}\right)\right)-\epsilon$, which is a contradiction.

B.4.2. Bidders with signals below $s_{z}^{p}$ In this part of the proof, we'll show that bidders with signals below $s_{z}^{p}$ don't have a profitable deviation to bidding above $b_{z}^{p}$ when $z$ is sufficiently large, using our restriction on the priors that $\rho(0)>\rho^{*}$. Remember that $s_{R}^{\kappa}$ is the signal such 
that $F\left(s_{R}^{\kappa} \mid R\right)=1-\kappa$. To prove that there is no profitable deviation, we will start with the following lemma:

LEMma $4 \quad \exists Z_{5} \in \mathbb{Z}$ such that $u\left(\rho_{z}^{+}\left(s, s_{z}^{p}\right)\right)-b_{z}^{p} \leq 0$ for every $s<s_{z}^{p}$, every $z>Z_{5}$.

PROOF: We'll make our argument under the assumption that the limit of the sequence $\left\{s_{z}^{p}, b_{z}^{p}\right\}_{z=1}^{\infty}=\left(s^{p}, b^{p}\right)$ exists and then we will verify this in lemma 5 . The next four claims are the steps of the proof.

CLAIM $1 \quad s^{p} \geq s_{R}^{\kappa}$

Proof: On the way to a contradiction, suppose that $s^{p}<s_{R}^{\kappa}$. Then, $\lim _{z \rightarrow \infty} \inf _{s>s_{z}^{p}} \rho_{z}^{+}\left(s, s_{z}^{p}\right)=$ 0 , because $\lim _{z} P\left(Y_{z-1}^{\kappa z} \leq s_{z}^{p} \mid L\right)=1$ and $\lim _{z} P\left(Y_{z-1}^{\kappa z} \leq s_{z}^{p} \mid R\right)=0$ if $s^{p}<s_{R}^{\kappa}$. This contradicts the assertion in Remark 10 that $\rho\left(s_{1}=s, Y_{z-1}^{\kappa z} \leq s_{z}^{p}\right) \geq \rho^{*}$ for $s>s_{z}^{p}$.

Claim 2 If $s^{p}>s_{R}^{\kappa}$, then $\exists Z_{3} \in \mathbb{Z}$ such that $u\left(\rho_{z}^{+}\left(s, s_{z}^{p}\right)\right)-b_{z}^{p} \leq 0$ for every $s<s_{z}^{p}$, every $z>Z_{3}$.

ProOF: To show that types $s<s_{z}^{p}$ don't want to deviate bidding above $b_{z}^{p}$ for large $z$, first note that since $F\left(s^{p} \mid \omega\right)>1-\kappa$ for $\omega \in \Omega$, we have $\lim _{z \rightarrow \infty} \rho\left(s_{1}=s, p_{z}=b_{z}^{p}\right)=\rho\left(s_{1}=s\right)$ for every $s \in[0,1]$. Since we assumed $\rho(0)>\rho^{*}$, it's also true that $\rho(s)>\rho^{*}$ for every $s \in[0,1]$. Now we will show that there is a $Z_{3}$ such that $u\left(\rho_{z}^{+}\left(s, s_{z}^{p}\right)\right) \leq b_{z}^{p}$ for all $s<s_{z}^{p}$ if $z>Z_{3}$. On the way to a contradiction suppose that $u\left(\rho_{z}^{+}\left(s_{z}, s_{z}^{p}\right)\right)>b_{z}^{p}$ for some $s_{z}<s_{z}^{p}$ for every $z$ that belongs to an unbounded subset of natural numbers. Since $\lim _{z \rightarrow \infty} \rho\left(s_{1}=\right.$ $\left.0, p_{z}=b_{z}^{p}\right)=\rho(0)>\rho^{*}$, we have that $u\left(\rho_{z}^{+}\left(s, s_{z}^{p}\right)\right)$ is increasing in $s$ for $z$ sufficiently large and $u\left(\rho_{z}^{+}\left(s^{\prime}, s_{z}^{p}\right)\right) \geq u\left(\rho_{z}^{+}\left(s_{z}, s_{z}^{p}\right)\right)$ for every $s^{\prime}>s_{z}$. There are two cases to consider: either $b_{z}^{p}=\inf _{s^{\prime}>s_{z}^{p}} u\left(\rho_{z}^{+}\left(s^{\prime}\right)\right)$ or $b_{z}^{p}=\inf _{s^{\prime}<s_{z}^{p}} u\left(\rho_{z}^{-}\left(s^{\prime}\right)\right)$.

If $b_{z}^{p}=\inf _{s^{\prime}>s_{z}^{p}} u\left(\rho_{z}^{+}\left(s^{\prime}\right)\right)$, then $b_{z}^{p} \geq u\left(\rho_{z}^{+}\left(s_{z}, s_{z}^{p}\right)\right)$, which is a contradiction to $u\left(\rho^{+}\left(s_{z}, s_{z}^{p}\right)\right)>$ $b_{z}^{p}$. If $b_{z}^{p}=\inf _{s^{\prime}<s_{z}^{p}} u\left(\rho_{z}^{-}\left(s^{\prime}\right)\right)$, then notice the property of $s_{z}^{p}$ that, $u\left(\rho_{z}^{-}\left(s^{\prime}\right)\right)>u\left(\rho_{z}^{+}\left(s^{\prime}\right)\right)$ for all $s^{\prime}<s_{z}^{p}$, and therefore $\inf _{s^{\prime}<s_{z}^{p}} u\left(\rho_{z}^{-}\left(s^{\prime}\right)\right) \geq \sup _{s^{\prime}<s_{z}^{p}}\left(\rho_{z}^{+}\left(s^{\prime}, s_{z}^{p}\right)\right) \geq u\left(\rho_{z}^{+}\left(s_{z}, s_{z}^{p}\right)\right)$. Hence, $b_{z}^{p} \geq u\left(\rho_{z}^{+}\left(s_{z}, s_{z}^{p}\right)\right)$ which is a contradiction to the definition of $s_{z}$ that $u\left(\rho_{z}^{+}\left(s_{z}, s_{z}^{p}\right)\right)>b_{z}^{p}$.

Claim 3 If $s^{p}=s_{R}^{\kappa}, \exists Z_{4} \in \mathbb{Z}$ such that $u\left(\rho_{z}^{+}\left(s, s_{z}^{p}\right)\right)-b_{z}^{p} \leq 0$ for every $s<s_{z}^{p}$, every $z>Z_{4}$.

ProOF: This is the case when prices may indeed reveal some information. We'll start by arguing that pooling bids converge to $u(0)$.

The crucial observation in this case is that $\lim _{z \rightarrow \infty} \operatorname{Pr}\left(\omega=L \mid p_{z}=b_{z}^{p}, 1\right.$ wins with $\left.b_{z}^{p}\right)=1$. The reason for the above limit calculation is the following. Fix an $\epsilon>0$. Then, $\lim _{z \rightarrow \infty} \operatorname{Pr}\left(Y_{z-1}^{(\kappa-\epsilon) z}>\right.$ 
$\left.s_{z}^{p} \mid \omega=R\right)=0$. Therefore, $\lim _{z \rightarrow \infty} \operatorname{Pr}\left(p_{z}=b_{z}^{p}, 1\right.$ wins with $\left.b_{z}^{p} \mid \omega=R\right) \leq \frac{\epsilon}{1-\kappa+\epsilon}$. Since this is true for every $\epsilon>0$, it has to be that

$$
\lim _{z \rightarrow \infty} \operatorname{Pr}\left(p_{z}=b_{z}^{p}, 1 \text { wins with } b_{z}^{p} \mid \omega=R\right)=0
$$

On the other side $\lim _{z \rightarrow \infty} \operatorname{Pr}\left(p_{z}=b_{z}^{p} \mid \omega=L\right)=1$ because $1-F\left(s_{R}^{\kappa} \mid \omega=L\right)<\kappa$. Therefore,

$$
\lim _{z \rightarrow \infty} \operatorname{Pr}\left(\omega=L \mid p_{z}=b_{z}^{p}, 1 \text { wins with } b_{z}^{p}\right)>0 .
$$

Combining equation 6 and inequality 7 delivers that

$$
\lim _{z \rightarrow \infty} \operatorname{Pr}\left(\omega=L \mid p_{z}=b_{z}^{p}, 1 \text { wins with } b_{z}^{p}\right)=1
$$

Along similar lines we argue that,

$$
\lim _{z \rightarrow \infty} \frac{\operatorname{Pr}\left(\omega=L \mid p_{z}=b_{z}^{p}, 1 \text { wins with } b_{z}^{p}\right)}{\operatorname{Pr}\left(\omega=L \mid p_{z}=b_{z}^{p}\right)}=\infty
$$

Now we'll finish the argument that pooling bids converge to $u(0)$. Our first observation is that $\lim _{z \rightarrow \infty} u\left(\rho_{z}^{-}\left(s, s_{z}^{p}\right)\right)=u(0)$, which follows from equality 8. Examining the way $b_{z}^{p}$ is set, we have

$$
\begin{aligned}
\max \left\{\inf _{s<s_{z}^{p}} u\left(\rho_{z}^{-}\left(s, s_{z}^{p}\right)\right), u\left(\rho_{z}^{-}\left(s_{z}^{p}\right)\right)\right\} & \\
& \geq b_{z}^{p} \\
& =\min \left\{\inf _{s<s_{z}^{p}} u\left(\rho_{z}^{-}\left(s, s_{z}^{p}\right)\right), \inf _{s>s_{z}^{p}} u\left(\rho_{z}^{+}\left(s, s_{z}^{p}\right)\right)\right\} \\
& \geq \min \left\{\inf _{s<s_{z}^{p}} u\left(\rho_{z}^{-}\left(s, s_{z}^{p}\right)\right), \inf _{s>s_{z}^{p}} u\left(\rho_{z}^{-}\left(s, s_{z}^{p}\right)\right)\right\} .
\end{aligned}
$$

Since both expressions in the last term and both expressions in the first term have limits equal to $u(0)$, by the Sandwich theorem, the limit of the pooling bids is $u(0)$, completing the argument.

Suppose that $\rho^{+}\left(s, s_{z}^{p}\right)<\rho^{*}$. Note that, there is an $\epsilon>0$ such that $\liminf _{z \rightarrow \infty} \rho^{+}\left(0, s_{z}^{p}\right)>\epsilon$. This is because, $\rho^{+}\left(s, s_{z}^{p}\right) \geq \rho^{*}$ for every $s>s_{z}^{p}$ and because of the limited individual information assumption on the signal distributions. Therefore, $u\left(\rho^{+}\left(s, s_{z}^{p}\right)\right)<u\left(\rho^{+}\left(0, s_{z}^{p}\right)\right)<$ $u(0)$. Since we have shown that $\lim _{z} b_{z}^{p}=u(0)$, when $z$ is sufficiently large, $u\left(\rho^{+}\left(s, s_{z}^{p}\right)\right)<b_{z}^{p}$, proving the claim for this case.

Suppose that $\rho^{+}\left(s, s_{z}^{p}\right) \geq \rho^{*}$ and suppose on the way to a contradiction that $u\left(\rho_{z}^{+}\left(s, s_{z}^{p}\right)\right)>$ $b_{z}^{p}$. It then follows that, $b_{z}^{p}=\inf _{s<s_{z}^{p}} u\left(\rho_{z}^{-}\left(s, s_{z}^{p}\right)\right)$. Then, since $u(\rho)$ is increasing in $\rho$ and since 
$\rho_{z}^{+}$is increasing in its first argument, there is an $\epsilon>0$ such that $u\left(\rho_{z}^{+}\left(s^{\prime}, s_{z}^{p}\right)\right)>b_{z}^{p}+\epsilon$ for every $s^{\prime} \geq s$. Since $\rho_{z}^{+}$is continuous in its second argument, then for some $s^{\prime \prime}<s_{z}^{p}$, $u\left(\rho_{z}^{+}\left(s^{\prime \prime}\right)\right)>b_{z}^{p}+\epsilon$. Since $u\left(\rho_{z}^{-}\left(s^{\prime \prime \prime}\right)\right)>u\left(\rho_{z}^{+}\left(s^{\prime \prime \prime}\right)\right)$ for every $s^{\prime \prime \prime}<s_{z}^{p}, u\left(\rho_{z}^{-}(s)\right)>b_{z}^{p}+\epsilon$ for every $s \in\left(s^{\prime \prime}, s_{z}^{p}\right)$. However, this contradicts that $b_{z}^{p}=\inf _{s<s_{z}^{p}} u\left(\rho_{z}^{-}\left(s, s_{z}^{p}\right)\right)=\inf _{s<s_{z}^{p}} u\left(\rho_{z}^{-}(s)\right)$. This completes the proof of the claim.

We have proven the lemma. Now we will argue that bidders with signals less than $s_{z}^{p}$ don't have profitable deviations to bid above $s_{z}^{p}$. We start by arguing that, for $s>s_{z}^{p}$, if $\rho\left(s^{\prime}, p_{z}=\right.$ $b(s)) \geq \rho^{*}$, and if $s^{\prime}<s$, then $u\left(\rho\left(s^{\prime}, p_{z}=b(s)\right)\right) \leq b(s)$. This is because, $u(\rho)$ is increasing in $\rho$ when $\rho \geq \rho^{*}$. Second, we observe that again if $s>s_{z}^{p}$ and if $\rho\left(s^{\prime}, p_{z}=b(s)\right)<\rho^{*}$, then $u\left(\rho\left(s^{\prime}, p_{z}=b(s)\right)\right)<u\left(\rho_{z}^{-}\left(s^{\prime}, s_{z}^{b}\right)\right.$. Since we have shown in the above lemma that for $s^{\prime}<s_{z}^{p}$, $u\left(\rho_{z}^{-}\left(s^{\prime}, s_{z}^{b}\right) \leq b_{z}^{p}\right.$, and since $b(s)>b_{z}^{p}$, we have that $u\left(\rho\left(s^{\prime}, p_{z}=b(s)\right)\right)<b(s)$. This proves that, bidders with types less than $s_{z}^{p}$ don't have a profitable deviation to any bid above $b_{z}^{p}$.

\section{Proof of Theorem 1}

Theorem 1. Suppose that Assumptions 1-4 hold. If Condition 1 and Condition 2 hold, then there exists an equilibrium sequence $\boldsymbol{b}$ which reveals no information.

PROOF: We will prove this theorem by using the same construction that we used to prove Theorem 2. Notice that the assumption that $\rho(0) \geq \rho^{*}$ facilitates that the hypothesis of Theorem 2 is satisfied, and hence the constructed bidding strategies constitute an equilibrium when $z$ is sufficiently large.

We will now show that if $u(0)>\liminf _{s>s_{R}^{\kappa}} u(\rho(s))$, then the limit of the cutoff types as $z$ goes to infinity, which we denote by $s^{p}$ is strictly larger than $s_{R}^{\kappa}$. The implication of this inequality is that equilibrium prices become the pooling bid in both states of the world with probabilities approaching one, and hence prices reveal no information as the market gets arbitrarily large.

We have already proven in the proof of theorem 2 that any limit point of the cutoffs has to be at least $s_{R}^{\kappa}$. Thus it remains to show that $s_{R}^{\kappa}$ is not a limit point of the cutoff types constructed in the sequence of bidding functions.

On the way to a contradiction, suppose our claim is not true, i.e., $s_{R}^{\kappa}$ is the limit point of the cutoff types. Then as we argued in the proof of theorem 2 , the pooling bid, $b_{z}^{p}$ goes to $u(0)$ as $z$ goes to $\infty$. On the other side, $\rho_{z}^{+}\left(s, s_{z}^{p}\right) \geq \rho^{*}$ for $s>s_{z}^{p}$ by Remark 10. Moreover, $\rho_{z}^{+}\left(s, s_{z}^{p}\right)<\rho(s)$. Therefore, $\inf _{s>s_{z}^{p}} u\left(\rho^{+}\left(s, s_{z}^{p}\right)\right)<\inf _{s>s_{z}^{p}} u(\rho(s))$ and $\inf _{s>s_{z}^{p}} u\left(\rho^{+}\left(s, s_{z}^{p}\right)\right) \geq$ $b_{z}^{p}$. If $s_{R}^{\kappa}$ is a limit point of $s_{z}^{p}$, then along the sequence where $s_{R}^{\kappa}$ is the limit of the sequence, we conclude that $\inf _{s>s_{R}^{\kappa}} u(\rho(s)) \geq \lim _{z \rightarrow \infty} b_{z}^{p}=u(0)$. This contradicts our initial hypothesis that $u(0)>\inf _{s>s_{R}^{\kappa}} u(\rho(s))$. 


\section{Proof of Lemma 1}

Lemma 1. Suppose that Assumptions 1-4 hold. Every equilibrium bidding function b that is nondecreasing satisfies the following conditions:

(i) There is a cutoff signal $s^{p} \in[0,1]$ and a pooling bid $b^{p}$ such that $b(s)=b^{p}$ for every $s<s^{p}$, and $b(s)>b^{p}$ for every $s>s^{p}$.

(ii) The bidding function $b(s)$ is strictly increasing in the range $\left(s^{p}, 1\right]$.

(iii) If $s^{p} \in(0,1)$, then the equilibrium action is $a\left(s, b(s), b^{p}\right)=r$ for $s>s^{p}$; and it is $a(s, b(s), p)=r$ for every $p \in\left(b^{p}, b(s)\right]$ for which there exists an $s^{\prime} \in S$ such that $b\left(s^{\prime}\right)=p$.

(iv) For $s \in\left[0, s^{p}\right), a\left(s, b(s), b^{p}\right)=l$.

PROOF: There are two cases to consider, either $b$ is strictly increasing or there is an atom in the bid distribution.

Case 1: If $b$ is strictly increasing, then the first part of the lemma is true by picking $s^{p}=0$. The second part of the lemma for this case claims that $b$ is a la ? (abbreviated as PS in the following). This is a slight modification of the arguments in PS, the second part of 'proof of proposition 1' in page 1272 .

Case 2: Suppose that the bid function has an atom at some bid $b^{p}$. Then the monotonicity of the bidding function implies that $b(s)=b^{p}$ for an interval of signals, $S\left(b^{p}\right)=\left(s^{\prime}, s^{p}\right)$ with $s^{\prime}<s^{p}$ and $b(s)=b^{p}$ for every $s \in S\left(b^{p}\right)$ and $b(s)>b^{p}$ for every $s>s^{p}$. In steps 1,2 and 3 below we will show that there can be at most one atom in the bid distribution and that $s^{\prime}=0$.

Step 1: The first step is to show that $\rho\left(s_{1}=s^{p}, p=b^{p}, 1\right.$ wins with $\left.b^{p}\right)<\rho^{*}$. On the way to a contradiction, suppose that it's not true. Then due to winner's and loser's curse (see PS, page 1272), types in $S\left(b^{p}\right)$ would deviate and bid slightly above $b^{p}$. This follows from the monotonicity of the bidding function $b$.

Step 2: We will now argue that

$$
\rho\left(s_{1}=s, p, 1 \text { wins with } b(s)\right)<\rho^{*}
$$

for every $s<s^{p}$ and $p \leq b(s)$. We first claim that the following is true for every $p^{\prime}<b^{p}$ which is in the range of $b$.

$$
\rho\left(s_{1}=s, p^{\prime}\right)<\rho\left(s_{1}=s, p=b^{p}, 1 \text { wins with } b^{p}\right) .
$$


This is a non-trivial claim and the proof is in Lemma 7. Moreover,

$$
\rho\left(s_{1}=s, p, 1 \text { wins with } p\right) \leq \rho\left(s_{1}=s, p\right) \text {. }
$$

This inequality is a standard argument from lemma 7 of PS, at page 1272. Combining the two inequalities in this step with the result in step 1 delivers the claim.

Step 3: We will now argue that all types below $s^{p}$ bid $b^{p}$.

On the way to a contradiction, assume that a positive measure of types bid strictly below $b^{p}$ and let $s^{\prime \prime}<s^{\prime}$ be such a type. By lemma 7 , the probability that type $s^{\prime \prime}$ puts on state $\mathrm{L}$ were she to bid $b^{p}$ and the price is any price between her bid and $b^{p}$ is weakly higher than that of types who are bidding $b^{p}$. Formally, for any $p^{\prime} \leq b^{p}$ that is in the range of $b$, the following holds:

$$
\operatorname{Pr}\left(\omega=L \mid s_{1}=s^{\prime \prime}, p^{\prime}, 1 \text { wins by bidding } b^{p}\right) \geq \operatorname{Pr}\left(\omega=L \mid s_{1}=s^{\prime}, p^{\prime}, 1 \text { wins by bidding } b^{p}\right) .
$$

Since bidding slightly below $b^{p}$ is a feasible strategy, we have that,

$$
u\left(\rho\left(s_{1}=s^{\prime}, b^{p}, 1 \text { wins by bidding } b^{p}\right)\right) \leq b^{p} .
$$

Therefore, $b^{p}$ is weakly less than the value of the object to types who bid $b^{p}$ conditional on the price being $b^{p}$ and they winning the object. Since this value is strictly less than the value when the price is strictly lower than $b^{p}$, these types make strictly positive profits when the price is strictly less than $b^{p}$. And finally, the bid of $s^{\prime \prime}$ cannot be an atom because the value of the object conditional on losing when the price is her bid is strictly larger than the value if the price was strictly above her bid but not higher than $b^{p}$, which contradicts her bid being an atom (This is a completely symmetric argument as lemma 7). More precisely, for any $p^{\prime} \in\left(b\left(s^{\prime \prime}\right), b^{p}\right]$ such that $b(s)=p^{\prime}$ for some $s \in\left(s^{\prime \prime}, s^{p}\right)$,

$\operatorname{Pr}\left(\omega=L \mid s_{1}=s^{\prime \prime}, p=b\left(s^{\prime \prime}\right), 1\right.$ loses by bidding $\left.b\left(s^{\prime \prime}\right)\right) \geq \operatorname{Pr}\left(\omega=L \mid s_{1}=s, p^{\prime}, 1\right.$ wins by bidding $\left.p^{\prime}\right)$

Therefore,

$$
\begin{aligned}
& u\left(\rho\left(s_{1}=s^{\prime \prime}, p=b\left(s^{\prime \prime}\right), 1 \text { loses by bidding } b\left(s^{\prime \prime}\right)\right)\right) \geq u\left(\rho\left(s_{1}=s, p^{\prime}, 1 \text { wins by bidding } p^{\prime}\right)\right) \geq \\
& p^{\prime}>b\left(s^{\prime \prime}\right) .
\end{aligned}
$$

Therefore, type $s^{\prime \prime}$ would have an incentive to bid strictly above $b\left(s^{\prime \prime}\right)$, yielding a contradiction to $b\left(s^{\prime \prime}\right)$ being an atom. Since $b\left(s^{\prime \prime}\right)$ is not an atom, $s^{\prime \prime}$ has a strict incentive to bid $b^{p}$, yielding the contradiction. 
Step 4: Now we consider bids above $b^{p}$ and will show that

$$
\rho\left(s_{1}=s^{p}, p=b^{p}\right)>\rho^{*}
$$

Since we have shown that there can be at most one atom, $b$ does not have a constant part above $s^{p}$. Therefore, it should be that $\rho\left(s_{1}=s^{p}, p=b^{p}\right)>\rho^{*}$. This follows from monotonicity of $b$ and the winner's curse. The reason is that otherwise signals lower and arbitrarily close to $s^{p}$ would have a provitable deviation to bid above $b^{p}$ (see PS, page 1272 again). Moreover $\rho\left(s_{1}=s, p=b\left(s^{\prime}\right)\right)>\rho^{*}$ for $s, s^{\prime}>s^{p}$ from Weak MLRP.

We now conclude that $b$ has to be a la PS for types above $s^{p}$, i.e., for $s>s^{p}, b(s)=$ $u\left(\rho\left(s_{1}=s, Y_{n-1}^{k}=s\right)\right)$. This follows from PS, because the value of the object is strictly increasing in the probability that the bidder assigns to state $R$ for types above $s^{p}$.

\section{E. Miscallenous Results}

LEMma 5 There is a unique signal $s^{*}$ which is a limit point of the cutoffs $\left\{s_{z}^{p}\right\}_{z \geq 0}$. Moreover either $s^{*}=s_{R}^{\kappa}$ or $s^{*}$ is the unique signal with the property that for $\bar{\rho}(s):=\rho(s) \frac{\kappa-(1-F(s \mid R)))}{F(s \mid R)} \frac{F(s \mid L)}{\kappa-(1-F(s \mid L)))}$, $u(\bar{\rho}(s))-u(\rho(s))<(>) 0$ for $s<s^{*}\left(s>s^{*}\right)$.

ProOF: Let $s^{*}$ be a limit point of the sequence, and rename the original sequence so that the limit is $s^{*}$. We have proven that $s^{*} \geq s_{R}^{\kappa}$ in claim 1 of proof of theorem 2 . So now assume that $s^{*}>s_{R}^{\kappa}$. We first note that, $\rho_{z}^{-}\left(s_{z}^{p}\right)$ can be more conveniently expressed by the following equality:

$$
\rho_{z}^{-}\left(s_{z}^{p}\right)=\rho\left(s_{z}^{p}\right) \frac{E\left[\frac{\rho z-\left(\text { number of bids } \geq s_{z}^{p}\right)}{z-\left(\text { number of bids } \geq s_{z}^{p}\right)} \mid R\right]}{E\left[\frac{\rho z-\left(\text { number of bids } \geq s_{z}^{p}\right)}{z-\left(\text { number of bids } \geq s_{z}^{p}\right)} \mid L\right]}
$$

Our first observation is that

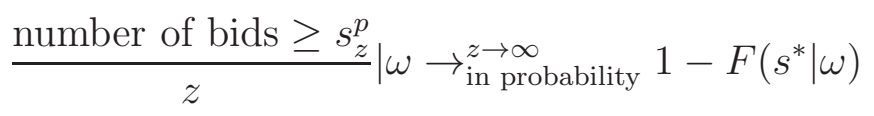

Therefore as $z \rightarrow \infty$,

$$
E\left[\frac{\rho z-\left(\text { number of bids } \geq s_{z}^{p}\right)}{z-\left(\text { number of bids } \geq s_{z}^{p}\right)} \mid \omega\right] \rightarrow \frac{\kappa-\left(1-F\left(s^{*} \mid \omega\right)\right)}{F\left(s^{*} \mid \omega\right)}
$$


and hence,

$$
\frac{E\left[\frac{\rho z-\left(\text { number of bids } \geq s_{z}^{p}\right)}{z-\left(\text { number of bids } \geq s_{z}^{p}\right)} \mid R\right]}{E\left[\frac{\rho z-\left(\text { number of bids } \geq s_{z}^{p}\right)}{z-\left(\text { number of bids } \geq s_{z}^{p}\right)} \mid L\right]} \rightarrow \frac{\frac{\kappa-\left(1-F\left(s^{*} \mid R\right)\right)}{F\left(s^{*} \mid R\right)}}{\frac{\kappa-\left(1-F\left(s^{*} \mid L\right)\right)}{F\left(s^{*} \mid L\right)}}
$$

Therefore as $z \rightarrow \infty$,

$$
\rho_{z}^{-}\left(s_{z}^{p}\right) \rightarrow \rho\left(s^{*}\right) \frac{\frac{\kappa-\left(1-F\left(s^{*} \mid R\right)\right)}{F\left(s^{*} \mid R\right)}}{\frac{\kappa-\left(1-F\left(s^{*} \mid L\right)\right)}{F\left(s^{*} \mid L\right)}}
$$

Since $s^{*}>s_{R}^{\kappa}$, as $z \rightarrow \infty$,

$$
\rho_{z}^{+}\left(s_{z}^{p}\right) \rightarrow \rho\left(s^{*}\right)
$$

Since each $s_{z}^{p}$ has the feature that $u\left(\rho_{z}^{-}(s)\right)-u\left(\rho_{z}^{+}(s)\right) \stackrel{(>)}{<} 0$ for $s \stackrel{(<)}{>} s_{z}^{p}$, and since for each $s>s_{R}^{\kappa}$,

$$
\rho_{z}^{-}(s) \rightarrow \rho(s) \frac{\frac{\kappa-(1-F(s \mid R))}{F(s \mid R)}}{\frac{\kappa-(1-F(s \mid L))}{F(s \mid L)}}
$$

we have that for $s \in\left(s_{R}^{\kappa}, 1\right)$,

$$
\Delta(s):=u\left(\rho(s)\left(\frac{\kappa-(1-F(s \mid R)))}{F(s \mid R)} \frac{F(s \mid L)}{\kappa-(1-F(s \mid L)))}\right)\right)-u(\rho(s)) \stackrel{(\geq)}{\leq} 0 \text { for } s \stackrel{(\leq)}{\geq} s^{*} .
$$

The term $\Delta(s)$ is strictly decreasing in the interval $\left(s_{R}^{\kappa}, 1\right)$ and is strictly negative when $s$ is close to 1 , therefore, there should be at most one signal $s^{*}$ that can be a limit point in the range $\left(s_{R}^{\kappa}, 1\right)$.

Now suppose that $s_{R}^{\kappa}$ is a limit point. We will show that no signal $s>s_{R}^{\kappa}$ can be a limit point. If $s_{R}^{\kappa}$ is a limit point of the sequence, then it should be that, for every $s>s_{R}^{\kappa}, \Delta(s)<0$.

However, then no $s>s_{R}^{\kappa}$ can be a limit point since this contradicts the previous finding that for a signal $s$ to be a limit point, it has to be that for signals $s^{\prime} \in\left(s_{R}^{\kappa}, s\right), \Delta(s)>0$.

Hence we have shown that if $s_{R}^{\kappa}$ is a limit point, then it is the unique limit point, and if it is not, and if an $s>s_{R}^{\kappa}$ is a limit point, then it is unique. This completes the argument that the sequence has a unique limit point.

LEMma 6 If $f(0 \mid L) \neq f(0 \mid R)$, then $\rho_{z}^{-}(s)>\rho_{z}^{+}(s)$ for any $s \in(0,1)$. Moreover both of these functions are strictly increasing in $s$. 
ProOF: The first claim in this lemma is identical to the argument in Lemma 7 in ?, page 1272 , and is called loser's curse.

The claim that $\rho_{z}^{+}(s)$ and $\rho_{z}^{-}(s)$ are strictly increasing is standard and follows from the MLRP assumption. Therefore the proof is omitted.

LEMMA 7 In an increasing equilibrium bidding function $b$, if there is an atom at bid $b^{p}$, then for any $p<b^{p}$ the following holds:

$$
\operatorname{Pr}\left(\omega=L \mid s_{1}=s, p\right)>\operatorname{Pr}\left(\omega=L \mid s_{1}=s, p=b^{p}, 1 \text { wins with } b^{p}\right) .
$$

Proof: Let the interval of types who are bidding at the atom bid be $\left(s^{\prime}, s^{\prime \prime}\right)$. Then $\operatorname{Pr}(\omega=$ $\left.L \mid s_{1}=s, p\right)>f\left(\omega=L \mid s_{1}=s\right) \operatorname{Pr}\left(\omega=L \mid Y_{n-1}^{k}=s^{\prime}\right)$. The term $\operatorname{Pr}\left(\omega=L \mid s_{1}=s, p=b^{p}, 1\right.$ wins with $b^{p}$ ) is calculated using the following steps:

$$
\begin{aligned}
1-F_{t}\left(s^{\prime}, s^{\prime \prime} \mid \omega\right) & :=\frac{F\left(s^{\prime \prime} \mid \omega\right)-F\left(s^{\prime} \mid \omega\right)}{F\left(s^{\prime \prime} \mid \omega\right)} \\
C_{j}^{n-1-i}(\omega) & :=\left(\begin{array}{c}
n-1-i \\
j
\end{array}\right)\left(1-F_{t}\left(s^{\prime}, s^{\prime \prime} \mid \omega\right)\right)^{j}\left(F_{t}\left(s^{\prime}, s^{\prime \prime} \mid \omega\right)\right)^{n-1-j} \\
D^{i}(\omega) & :=\left(\begin{array}{c}
n-1 \\
i
\end{array}\right)\left(1-F\left(s^{\prime \prime} \mid \omega\right)\right)^{i}\left(F\left(s^{\prime \prime} \mid \omega\right)\right)^{n-1-i} \sum_{n-1-i \geq j \geq k-i} C_{j}^{n-1-i}(\omega) \frac{k-i}{j+1} \\
\operatorname{Pr}\left(s_{1}\right. & \left.=s, p=b^{p}, 1 \text { wins with } b^{p} \mid \omega\right)=f(s \mid \omega) \sum_{0 \leq i \leq k-1} D^{i}(\omega) \\
\operatorname{Pr}(\omega & \left.=L \mid s_{1}=s, p=b^{p}, 1 \text { wins with } b^{p}\right)=\frac{f(s \mid L) \sum_{0 \leq i \leq k-1} D^{i}(L)}{f(s \mid R) \sum_{0 \leq i \leq k-1} D^{i}(R)}
\end{aligned}
$$

Explanation: The probability that 1 wins with $b^{p}$, the price is $b^{p}$ conditional on $\omega$ can be calculated as the sum of the probabilities of winning in each of the following events, $w^{i, j}$ where $i \leq k-1$ bidders bid above $s^{\prime \prime}$, and $k-i \leq j \leq n-1-i$ bidders bid the pooling bid. The probability of winning conditional on event $w^{i, j}$ is $\frac{k-i}{j+1}$, since there are $k-i$ objects remaining for the $j+1$ bidders bidding the pooling bid. The above expressions calculate the probability of each event $w^{i, j}$ in each state and calculate the total winning probability in each state.

Similarly the term $f\left(\omega=L \mid s_{1}=s\right) \operatorname{Pr}\left(\omega=L \mid Y_{n-1}^{k}=s^{\prime}\right)$ is calculated using the following 
steps:

$$
\begin{gathered}
\operatorname{Pr}\left(Y_{n-1}^{k}=s^{\prime} \mid \omega\right)=\left(\begin{array}{c}
n-1 \\
1
\end{array}\right) f\left(s^{\prime} \mid \omega\right) \sum_{0 \leq i \leq k-1}\left(\begin{array}{c}
n-2 \\
i
\end{array}\right)\left(1-F\left(s^{\prime \prime} \mid \omega\right)\right)^{i}\left(F\left(s^{\prime \prime} \mid \omega\right)\right)^{n-2-i} C_{k-i-1}^{n-2-i}(\omega) \\
f\left(\omega=L \mid s_{1}=s\right) \operatorname{Pr}\left(\omega=L \mid Y_{n-1}^{k}=s^{\prime}\right)=\frac{f(s \mid L) \operatorname{Pr}\left(Y_{n-1}^{k}=s^{\prime} \mid L\right)}{f(s \mid R) \operatorname{Pr}\left(Y_{n-1}^{k}=s^{\prime} \mid R\right)} .
\end{gathered}
$$

We will now show the following

$$
\frac{f(s \mid L) \operatorname{Pr}\left(Y_{n-1}^{k}=s^{\prime} \mid L\right)}{f(s \mid R) \operatorname{Pr}\left(Y_{n-1}^{k}=s^{\prime} \mid R\right)}>\frac{f(s \mid L) \sum_{0 \leq i \leq k-1} D^{i}(L)}{f(s \mid R) \sum_{0 \leq i \leq k-1} D^{i}(R)}
$$

or equivalently the following,

$$
\frac{\left(\begin{array}{c}
n \\
1
\end{array}\right) f\left(s^{\prime} \mid L\right) \sum_{0 \leq i \leq k-1}\left(\begin{array}{c}
n-2 \\
i
\end{array}\right)\left(1-F\left(s^{\prime \prime} \mid L\right)\right)^{i}\left(F\left(s^{\prime \prime} \mid L\right)\right)^{n-2-i} C_{k-i-1}^{n-2-i}(L)}{\left(\begin{array}{c}
n \\
1
\end{array}\right) f\left(s^{\prime} \mid R\right) \sum_{0 \leq i \leq k-1}\left(\begin{array}{c}
n-2 \\
i
\end{array}\right)\left(1-F\left(s^{\prime \prime} \mid R\right)\right)^{i}\left(F\left(s^{\prime \prime} \mid R\right)\right)^{n-2-i} C_{k-i-1}^{n-2-i}(R)}>\frac{\sum_{0 \leq i \leq k-1} D^{i}(L)}{\sum_{0 \leq i \leq k-1} D^{i}(R)} .
$$

We define the following notation before we proceed:

$$
E^{i}(\omega):=\left(\begin{array}{c}
n-1 \\
1
\end{array}\right) f\left(s^{\prime} \mid \omega\right)\left(\begin{array}{c}
n-2 \\
i
\end{array}\right)\left(1-F\left(s^{\prime \prime} \mid \omega\right)\right)^{i}\left(F\left(s^{\prime \prime} \mid \omega\right)\right)^{n-2-i} C_{k-i-1}^{n-2-i}(\omega)
$$

We first present the following identity which requires minor algebra:

$$
D^{i}(\omega)=E^{i}(\omega) \frac{\left(1-F_{t}\left(s^{\prime}, s^{\prime \prime} \mid \omega\right)\right) F\left(s^{\prime \prime} \mid \omega\right)}{f\left(s^{\prime} \mid \omega\right)} \sum_{k-i \leq j \leq n-i-1} \frac{(k-i) !(n-k-1) !}{(j+1) !(n-j-i-1) !}\left(\frac{1-F_{t}\left(s^{\prime}, s^{\prime \prime} \mid \omega\right)}{F_{t}\left(s^{\prime}, s^{\prime \prime} \mid \omega\right)}\right)^{j+i-k} .
$$

A second simplification of the above identity via a change of variables by letting $u:=$ $j-k+i$ delivers the following:

$$
D^{i}(\omega)=E^{i}(\omega) \frac{\left(1-F_{t}\left(s^{\prime}, s^{\prime \prime} \mid \omega\right)\right) F\left(s^{\prime \prime} \mid \omega\right)}{f\left(s^{\prime} \mid \omega\right)} \sum_{0 \leq u \leq n-k-1} \frac{(k-i) !(n-k-1) !}{(k-i+u+1) !(n-k-u-1) !}\left(\frac{1-F_{t}\left(s^{\prime}, s^{\prime \prime} \mid \omega\right)}{F_{t}\left(s^{\prime}, s^{\prime \prime} \mid \omega\right)}\right)^{u} .
$$

We first observe that the following inequality is satisfied:

$$
\frac{\left(1-F_{t}\left(s^{\prime}, s^{\prime \prime} \mid L\right)\right) F\left(s^{\prime \prime} \mid L\right)}{f\left(s^{\prime} \mid L\right)}<\frac{\left(1-F_{t}\left(s^{\prime}, s^{\prime \prime} \mid R\right)\right) F\left(s^{\prime \prime} \mid R\right)}{f\left(s^{\prime} \mid R\right)}
$$

Our second observation is that for any fixed $u \in\{0, \ldots, n-k-1\}$, the term $\frac{(k-i) !(n-k-1) !}{(k-i+u+1) !(n-k-u-1) !}$ is strictly increasing in $i$. 
Our third observation is that the following inequality holds for any $u$ :

$$
\left(\frac{1-F_{t}\left(s^{\prime}, s^{\prime \prime} \mid L\right)}{F_{t}\left(s^{\prime}, s^{\prime \prime} \mid L\right)}\right)^{u}<\left(\frac{1-F_{t}\left(s^{\prime}, s^{\prime \prime} \mid R\right)}{F_{t}\left(s^{\prime}, s^{\prime \prime} \mid R\right)}\right)^{u} .
$$

This follows from the MLRP assumption.

Our final observation is that $\frac{E^{i}(L)}{E^{i}(R)}$ is strictly increasing in $i$. This observation also follows from the MLRP assumption and a simple calculation.

These four observations yield the desired result.

\section{REFERENCES}

Bester, H. (1985): "Screening vs. rationing in credit markets with imperfect information," The American Economic Review, 75, 850-855.

- (1987): "The role of collateral in credit markets with imperfect information," European Economic Review, 31, 887-899.

Bond, P. And H. ERAslan (2010): "Information-based trade," Journal of Economic Theory, 145, 16751703.

CRIPPs, M. And J. Swinkels (2006): "Efficiency of large double auctions," Econometrica, 74, 47-92.

Golosov, M., G. Lorenzoni, And A. Tsyvinski (2011): "Decentralized Trading with Private Information," Working paper, Princeton University.

Grossman, S. (1981): "An introduction to the theory of rational expectations under asymmetric information," The Review of Economic Studies, 48, 541-559.

Grossman, S. And J. Stiglitz (1976): "Information and competitive price systems," The American Economic Review, 66, 246-253.

- (1980): "On the impossibility of informationally efficient markets," The American Economic Review, 70, 393-408.

HAYEK, F. (1945): "The use of knowledge in society," The American Economic Review, 35, 519-530.

Hong, H. AND M. Shum (2004): "Rates of information aggregation in common value auctions," Journal of Economic Theory, 116, 1-40.

JaCKSON, M. AND I. Kremer (2007): "On the informational inefficiency of discriminatory price auctions," Journal of Economic Theory, 132, 507-517.

Kremer, I. (2002): "Information aggregation in common value auctions," Econometrica, 70, 1675-1682.

Kremer, I. AND A. Skrzypacz (2005): "Information Aggregation and the Information Content of Order Statistics," Working paper, Stanford University.

Lauermann, S. (2007): "Dynamic Matching and Bargaining Games: A General Approach," University of Michigan mimeo.

Lauermann, S. And A. Wolinsky (2011): "Search with Adverse Selection," Working Paper, Northwestern University.

- (2012): "A Common Value Auction with Bid Solicitation," Working Paper, Northwestern University.

Milgrom, P. (1979): "A convergence theorem for competitive bidding with differential information," Econometrica, 679-688.

Milgrom, P. And R. Weber (1982): "A theory of auctions and competitive bidding," Econometrica, 50, 1089-1122. 
(1985): "Distributional strategies for games with incomplete information," Mathematics of Operations Research, 619-632.

Osborne, M. And A. Rubinstein (2010): Bargaining and Markets, Academic Press.

Ostrovsky, M. (2009): "Information Aggregation in Dynamic Markets with Strategic Traders," Working paper, Stanford University.

Pesendorfer, W. And J. Swinkels (1997): "The loser's curse and information aggregation in common value auctions," Econometrica, 65, 1247-1281.

- (2000): "Efficiency and information aggregation in auctions," American Economic Review, 90, 499525 .

Reny, P. ANd M. Perry (2006): "Toward a strategic foundation for rational expectations equilibrium," Econometrica, 74, 1231-1269.

Rostek, M. And M. Weretka (2010): "Price Inference in Small Markets," Econometrica.

Rubinstein, A. And A. Wolinsky (1985): "Equilibrium in a market with sequential bargaining," Econometrica, 53, 1133-1150.

- (1990): "Decentralized trading, strategic behaviour and the Walrasian outcome," The Review of Economic Studies, 57, 63.

Stiglitz, J. And A. Weiss (1981): "Credit rationing in markets with imperfect information," The American Economic Review, 71, 393-410.

VIVES, X. (2011): "Strategic supply function competition with private information," Econometrica, 79, 1919-1966.

Wilson, R. (1977): "A bidding model of perfect competition," The Review of Economic Studies, 44, 511-518. 